\title{
Electron-nuclear correlations for photo-induced dynamics in molecular dimers
}

\author{
Dmitri S. Kilin, Yuri V. Pereversev, and Oleg V. Prezhdo* \\ University of Washington, Seattle WA, 98195
}

Submitted to J. Chem. Phys. November 6, 2018

\begin{abstract}
Ultrafast photoinduced dynamics of electronic excitation in molecular dimers is drastically affected by the dynamic reorganization of inter- and intra- molecular nuclear configuration modeled by a quantized nuclear degree of freedom [Cina et. al, J. Chem Phys. 118, 46 (2003)]. The dynamics of the electronic population and nuclear coherence is analyzed by solving the chain of coupled differential equations for population inversion, electron-vibrational correlation, etc. [Prezhdo, Pereverzev, J. Chem. Phys. 1136557 (2000)]. Intriguing results are obtained in the approximation of a small change of the nuclear equilibrium upon photoexcitation. In the limiting case of resonance between the electronic energy gap and the frequency of the nuclear mode these results are justified by comparison to the exactly solvable Jaynes-Cummings model. It is found that the photoinduced processes in the model dimer are arranged according to their time scales: (i) fast scale of nuclear motion, (ii) intermediate scale of dynamical redistribution of electronic population between excited states as well as growth and dynamics of electron-nuclear correlation, (iii) slow scale of electronic population approach to the quasi-equilibrium distribution, decay of electron-nuclear correlation, and decrease of the amplitude of mean coordinate oscillation. The latter processes are accompanied by a noticeable growth of the nuclear coordinate dispersion associated with the overall nuclear wavepacket width. The demonstrated quantum relaxation features of the photoinduced vibronic dynamics in molecular dimers are obtained by a simple method, applicable to systems with many degrees of freedom.
\end{abstract}

PACS numbers: 02.30.Jr, 05.10.Gg, 31.50.Gh, 82.20.Kh, 89.30.Cc

\footnotetext{
* Corresponding author. E-mail: prezhdo@u.washington.edu.
} 


\section{INTRODUCTION}

Recent achievements of femtosecond spectroscopy ${ }^{1}$ open new windows into the world of electron and exciton transfer dynamics in molecular systems, which play dominant roles in a variety of problems in physics, technology, atmospheric photochemistry, and biology $2,3,4,5,6$. Electron and exciton transfer drive formation and breaking of chemical bonds, corrosion reactions, ion tunneling microscopy, enzymatic activity in living cells, energy storage by adenosine triphosphate and harvesting of solar energy. For instance, the harvesting and storage of solar energy in the natural photosynthetic complexes, such as bacteriochlorophyll ${ }^{7}$ or Rhodobacter sphaeroids ${ }^{2}$, rely on the specific alignments of the light-harvesting units that favor efficient light absorption and subsequent energy transfer. The natural and artificial light harvesting complexes (LHC) ${ }^{8}$ involve exciton transfer of the primary photo-excitation in the antennae complex ${ }^{9}$, followed by a subsequent electron transfer that takes place in the reaction center of the natural LHC and porphyrin arrays of artificial $\mathrm{LHC}^{10,11}$.

The dynamics of electron and energy transfer, and formation of entangled electron-phonon states are investigated by a wide arsenal of femtosecond experimental techniques, which by application of one or several short laser pulses, provide time resolved information on couplings, correlations and states of molecular aggregates ${ }^{12,13,14,15,16,17}$. The multi-pulse time resolved techniques cover various frequency ranges, including X-ray, visible and infrared, and serve for determination of population, location and phase of electron-nuclear states of molecular systems. Thus, a recently developed Raman X-ray spectroscopy follows electronic state dynamics ${ }^{18}$. Laser pulses in the infrared diapason are used to investigate populations and mutual correlations of vibrational modes ${ }^{19,20}$. Visible frequency short laser pulses are applied to measure electronic state populations and state-to-state correlations ${ }^{21}$. Positions of nuclear wavepackets are detected by pump-probe spectroscopic techniques ${ }^{1}$. Relative phases

of electron-nuclear wavepackets of molecular aggregates are obtained using the nonlinear wavepacket interferometry ${ }^{22,23,24,25}$.

Theoretical methods describing exciton and electron transfer dynamics appeal, in one way or another, to both electronic and nuclear degrees of freedom ${ }^{26,27,28}$ and most commonly in chemical physics employ the notion of a reaction coordinate ${ }^{29}$. The reaction coordinate quantifies changes in the electronic states due to nuclear mode dynamics and is based on the fact that transfer is most effective for specific configurations of the nuclear subsystem. 
These nuclear configurations correspond to crossings of the potential surfaces and match the Franck-Condon window for the source and target states. In many cases, particularly for large systems, the nuclear subsystem leaves and never returns to the optimal configuration region due to stochastic noise and destructive interference among nuclear modes of different frequencies involved in the dynamics. This dynamic mismatch and dephasing of the nuclear modes is responsible for relaxation and decoherence ${ }^{30}$ in the exciton and electron transfer dynamics.

The coupled electron-phonon dynamics can be described by a number of theoretical approaches developed in chemical physics ${ }^{31}$, including the Gaussian wave-packet approaches $^{32,33}$, the semiclassical approximations ${ }^{34}$ based on Feynman path integrals ${ }^{35}$, and non-adiabatic molecular dynamics ${ }^{36,37,38}$. The quantum-classical mean-field approach and its multiconfiguration mean-field and surface hopping extensions are applied to a wide range of problems, including gas-phase scattering, surface ${ }^{5}$, solution and biological chemistry ${ }^{39}$. Semiempirical approaches introduce quantum corrections to classical mechanics. The recently developed Quantized Hamilton Dynamics (QHD) method $30,40,41,42,43,44$ offers a reduced description of quantum nuclear motion by disentangling coordinate, momentum, dispersion and higher order variables for each nuclear mode. A many body closure ${ }^{45}$ terminates the infinite chain of coupled equations reducing the number of equations and saving the calculation time. While the semiclassical and mean-field approaches are very effective from the numerical point of view, they provide approximate solutions. In particular, the mean-field approaches typically cannot describe the dynamic creation of superposition and entanglement between states leading to branching of nuclear dynamics.

Theoretical models that are able to describe the superposition and branching feature of coupled electron-phonon dynamics are available in the solid-state theory ${ }^{46}$ and quantum optics theories of atom-field interactions ${ }^{47}$. The quantum optics approaches to the description of photon modes coupled to atomic quantum states ${ }^{48}$ can be adopted to study electronphonon states in chemical, biological and nano-systems. A simple eigenstate solution for the correlated dynamics of two electronic states of an atom (fermion degree of freedom) coupled to a laser mode (boson degree of freedom), presented in 1963 by Jaynes and Cummings ${ }^{49,50}$, takes an important place among the optical methods. The Jaynes-Cummings solution was further developed by the operator algebra methods ${ }^{51}$. Known are the operator solutions for the lowering and raising operators ${ }^{52}$ and for the electronic state population ${ }^{53}$ that shows 
collapses $^{54}$ and revivals ${ }^{55}$. Interesting expressions are found for the uncertainty relationships for the operators describing the two-level atom interacting with a photon field ${ }^{56}$. The generalized operator solutions lead to a well-developed formalism in the Fokker-Planck equation formulation $^{57}$. The collapses and revivals in the two-level atom excitation dynamics of the Jaynes-Cummings model represent the simplest case of quantum relaxation common for exciton and electron transfer in molecular complexes ${ }^{58,59,60}$.

The quantum optics and QHD approaches are combined in this paper for the description of molecular aggregates, whose photoexcitation dynamics is strongly affected by dynamic rearrangements of vibrational degrees of freedom. The combination of methods is very effective for the calculation of the relaxation behavior of exciton transfer in molecular aggregates. Extending the original QHD approach that focuses onto semiclassical dynamics of nuclear modes, a QHD approximation is developed in present for the dynamics of the coupled electronic and nuclear variables, concentrating on the difference of the electronic state populations, i.e., population inversion. The resulting equations are very simple to be applied to large condensed phase chemical systems. Without reference to a thermal bath, as typical in the quantum relaxation theory, the QHD approximation for the dynamics of the Jaynes-Cummings model shows quantum relaxation features. The relaxation character of the exciton and electron transfer dynamics arises naturally in our approach due to destructive interference of quantum states, technically similar to quantum beats. The complex dynamics of the electron population in the Jaynes-Cummings model is made more understandable by a time-scale hierarchy ${ }^{61,62}$ of the relevant dynamical processes.

The paper is organized as follows. The relevant theoretical tools are introduced in Section II. The calculated quantum dynamics are analyzed in Section III, including a detailed discussion of the vibronic wavepacket. Section IV considers approach of the system to a state with large uncertainty. The physical processes in the system are organized in accordance with their characteristic times in Section V. The scenarios of experiments that can be described by the current method, and where the dynamical features found in this paper can play a dominant role, are discussed in Section VI. Finally, Section VII summarizes the main conclusions of this work. 


\section{MODEL}

Consider dynamics of electronic states in a molecular system. Typically, the initial populations of the states are derived from a thermal equilibrium. An external perturbation such as a femtosecond optical pulse quickly, within $10^{-15}-10^{-14} \mathrm{~s}$ breaks the equilibrium by enhancing population of excited states. The non-equilibrium populations evolve in time. The populations of the excited states are redistributed within an intermediate time interval of a few picoseconds, $10^{-12} \mathrm{~s}$. The population of the electronic states dephases due to interaction with inter and intramolecular vibrational modes. On a relatively long time scale of nanoseconds, $10^{-9} \mathrm{~s}$, the molecular system is de-excited by spontaneous emission induced by interaction with zeroth order vacuum oscillations of photon modes, or by some other mechanism. Upon electronic de-excitation, additional electron-vibration dynamics returns the system to thermal equilibrium.

The time-dependent processes that occur shortly after pumping are of interest in the present work. The focus is on dynamics of a pair of single-excitation localized electronic states $i=0,1$. One of the states $i=1$ is pumped initially and is coupled to and exchanges population with the second state. The two electronic states will be described in terms of the second quantization operators, creating $c_{i}^{+}$, or annihilating $c_{i}$ the excitation of localized state $i$. The Hamiltonian describing the picosecond $t \simeq 10^{-12} \mathrm{~s}$ dynamics of the excited states can be written in terms of the creation and annihilation operators as

$$
H_{0}=\sum_{i} \epsilon_{i} c_{i}^{+} c_{i}+\sum_{i} \sum_{j} J_{i j} c_{i}^{+} c_{j}
$$

The first term contains the electronic state energy $\epsilon_{i}$ times the operator of number of quanta, whose expectation value equals one $\left\langle c_{i}^{+} c_{i}\right\rangle=1$ if state $i$ is populated and zero if state $i$ is empty. The second term describes the coupling between the electronic states by removing population in state $j, c_{j}$ and creating population in state $i, c_{i}^{+}$with the coupling constant $J_{i j}$. In general, both energy and coupling depend on nuclear configuration. For small fluctuations of the nuclear coordinate $q$ the dependence of energy and coupling on $q$ can be well represented by the zeroth and first order terms in the Taylor series expansion

$$
\epsilon_{i}(q)=\epsilon_{i}^{(0)}+\left.q \frac{\partial \epsilon_{i}}{\partial q}\right|_{q=q_{i}}+\ldots, i=0,1
$$

Generally, the expansion coefficients are specific for each electronic state. The Taylor expansions of the coupling constants may also be different for the forward and backward 
transitions

$$
\begin{aligned}
& J_{01}=J_{01}^{(0)}+\left.q \frac{\partial J_{01}}{\partial q}\right|_{q=q_{0}}+\ldots \\
& J_{10}=J_{10}^{(0)}+\left.q \frac{\partial J_{10}}{\partial q}\right|_{q=q_{1}}+\ldots
\end{aligned}
$$

Typically, however, the coupling constants are identical for both directions $J_{01}=J_{10}$, $\partial J_{01} / \partial q=\partial J_{10} / \partial q$. The position independent terms in the expansion of the coupling constant can be eliminated by diagonalizing of the $q$-independent part of the electronic Hamiltonian.

The evolution of the nuclear coordinate $q$ augments the original electronic Hamiltonian (1) with a vibrational term, which equals $p^{2} /(2 m)+m \omega^{2} q^{2} / 2$ in the harmonic approximation. The vibrational Hamiltonian is the same for both electronic states. Multiplication of the vibrational Hamiltonian $\left(p^{2} /(2 m)+m \omega^{2} q^{2} / 2\right) \hat{\mathbf{1}}_{\text {el }}$ by a unit operator in the electronic subspace $\hat{\mathbf{1}}_{\mathrm{el}}=\sum_{i} c_{i}^{+} c_{i}$ leads to the following electron-phonon Hamiltonian

$$
H=\sum_{i} c_{i}^{+} c_{i}\{\epsilon_{i}^{(0)}+\underbrace{q \cdot \frac{\partial \epsilon_{i}}{\partial q}}+\frac{p^{2}}{2 m}+\underbrace{\frac{m \omega^{2} q^{2}}{2}}\}+\sum_{i} \sum_{j} c_{i}^{+} c_{j}\left\{q \cdot \frac{\partial J_{i j}}{\partial q}\right\}
$$

Consider the coordinate-dependent terms indicated in (4) by the underbrace sign in more detail. The equilibrium position $q_{i}$ for the harmonic motion correlated with the electronic state $i$ can be obtained explicitly by completing the square:

$$
\begin{aligned}
\epsilon_{i}^{\prime} \cdot q+\frac{m \omega^{2}}{2} q^{2} & =\frac{m \omega^{2}}{2}\{\underbrace{\epsilon_{i}^{\prime} q \frac{2}{m \omega^{2}}+q^{2}-\left(\frac{\epsilon_{i}^{\prime}}{m \omega^{2}}\right)^{2}}+\left(\frac{\epsilon_{i}^{\prime}}{m \omega^{2}}\right)^{2}\} \\
& =\frac{m \omega^{2}}{2}\left(q-q_{i}\right)^{2}+\frac{\left(\epsilon_{i}^{\prime}\right)^{2}}{2 m \omega^{2}},
\end{aligned}
$$

where $\epsilon_{i}^{\prime} \equiv \partial \epsilon_{i} / \partial q, E_{i}=\epsilon_{i}^{(0)}+\left(\epsilon_{i}^{\prime}\right)^{2} / 2 m \omega^{2}$, is the renormalized state-specific electronic energy and $q_{i}=\epsilon_{i}^{\prime}\left(m \omega^{2}\right)^{-1}$. The electron-phonon Hamiltonian takes the form

$$
H=\sum_{i} c_{i}^{+} c_{i}\left\{E_{i}+\frac{m \omega^{2}}{2}\left(q-q_{i}\right)^{2}\right\}+\sum_{i} \sum_{j} c_{i}^{+} c_{j}\left\{q \cdot \frac{\partial J}{\partial q}\right\}
$$

Without loss of generality $q_{0}=0, q_{1} \neq 0$. The Hamiltonian describing two electronic states coupled through a vibrational mode contains two harmonic potential energy surfaces 
corresponding to the two electronic states and centered at 0 and $q_{1}$. The states are coupled via a coordinate-dependent term similar to the Holstein Hamiltonian ${ }^{63}$

$$
\begin{aligned}
H= & c_{0}^{+} c_{0}[\underbrace{E_{0}+m \omega^{2} / 2 q^{2}}_{\text {lower potential }}+p^{2} / 2 m]+c_{1}^{+} c_{1}[\underbrace{E_{1}+m \omega^{2} / 2\left(q-q_{1}\right)^{2}}_{\text {upper potential }}+p^{2} / 2 m] \\
& +\underbrace{\left[c_{0}^{+} c_{1}+c_{1}^{+} c_{0}\right]}_{\text {transfer }} \cdot q \cdot \frac{\partial J}{\partial q}
\end{aligned}
$$

A two-level system of single excitation states is conveniently represented as a spin-1/2 particle in terms of three spin-projection operators $\hat{S}_{\mathrm{x}}, \hat{S}_{\mathrm{y}}, \hat{S}_{\mathrm{z}}$, which can be expressed using the lowering and raising operators $\hat{S}_{\mathrm{x}}=1 / 2\left(S_{+}+S_{-}\right), \hat{S}_{\mathrm{y}}=i / 2\left(S_{+}-S_{-}\right), \hat{S}_{\mathrm{z}}=$ $1 / 2\left(S_{+} S_{-}-S_{-} S_{+}\right)$.

$$
\begin{aligned}
S_{ \pm} & =S_{\mathrm{x}} \pm i S_{\mathrm{y}}, \\
S_{ \pm} S_{\mp} & =1 / 2 \pm S_{\mathrm{z}},
\end{aligned}
$$

The unique relationships between the spin-1/2 raising and lowering operators and the creation and annihilation operators of the electronic system in the language of second quantization are given by

$$
\begin{aligned}
c_{0}^{+} c_{0} & \equiv|0\rangle\langle 0| \equiv S_{-} S_{+}, \\
c_{1}^{+} c_{1} & \equiv|1\rangle\langle 1| \equiv S_{+} S_{-}, \\
c_{1}^{+} c_{0} & \equiv|1\rangle\langle 0| \equiv S_{+}, \\
c_{0}^{+} c_{1} & \equiv|0\rangle\langle 1| \equiv S_{-} .
\end{aligned}
$$

The evolution of the vibrational coordinate and momentum relevant for experiments occurs on an atomic scale demanding a quantum mechanical treatment. The quantum coordinate $q$ and momentum $p$ operators are conveniently replaced by the harmonic raising $a^{+}$and lowering $a$ operators

$$
\begin{aligned}
& q=\sqrt{\frac{\hbar}{2 m \omega}}\left(a^{+}+a\right), \\
& p=i \sqrt{\frac{\hbar m \omega}{2}}\left(a^{+}-a\right) .
\end{aligned}
$$

The above operator transformations translate the problem into the second quantization language of raising and lowering operators for both the electronic and vibrational degrees 
of freedom. The relevant dynamics will be represented by product operators containing spin and harmonic raising and lowering terms. The product operators describe resonance exchange of quanta between vibrational and electronic subsystems. The shift in the equilibrium coordinates of the two harmonic potentials is represented in the second quantization language by the dimensionless parameter $\bar{\alpha}=q \sqrt{m \omega / 2 \hbar}+i p \sqrt{\hbar / 2 m \omega}$, related to the original coordinate shift

$$
q=\sqrt{\frac{\hbar}{2 m \omega}}\left(\bar{\alpha}+\bar{\alpha}^{*}\right)
$$

The zero of electronic energy is chosen so that the initially unoccupied lower state $i=0$ has zero energy. The physically relevant electronic energy gap $\hbar \Omega=E_{1}-E_{0}$ is independent of energy origin. With all above changes, the electron-phonon Hamiltonian reads

$$
\begin{aligned}
H= & \hbar \omega S_{-} S_{+}\left[a^{+} a+1 / 2\right]+\hbar \omega S_{+} S_{-}\left[\left(a^{+}+\bar{\alpha}^{*}\right)(a+\bar{\alpha})+1 / 2\right] \\
& +g\left(S_{+}+S_{-}\right)\left(a^{+}+a\right)+S_{+} S_{-}\left[\hbar \Omega-\hbar \omega \bar{\alpha}^{*} \bar{\alpha}\right]
\end{aligned}
$$

where $g=\sqrt{\hbar / 2 m \omega} \quad \partial J / \partial q$.

The Hamiltonian (12) represents a dimer with a pair of electronic states $|0\rangle$ and $|1\rangle$. The states are separated by energy difference $\hbar \Omega$ and are coupled to a single harmonic vibrational mode. The equilibrium positions of the potential energy surfaces describing the vibrational mode in the two electronic states are shifted with respect to each other by $q_{1}$, specified by the dimensionless nuclear reorganization parameter $\bar{\alpha}$, as illustrated in Fig. 1This model is known as the Marcus model ${ }^{26}$ that has an enormous range of applications to exciton, electron, proton transfer and many other chemical reactions ${ }^{27,29,31}$.

In the limit of small nuclear reorganization the electron-phonon Hamiltonian transforms into a simpler form, known in quantum optics as the Jaynes-Cummings Hamiltonian ${ }^{49,50}$.

$$
H_{\mathrm{JCM}}=\omega\left(a^{+} a+1 / 2\right)+\Omega S_{+} S_{-}+g\left(a^{+}+a\right)\left(S_{+}+S_{-}\right) .
$$

Here and below $\hbar=1$. The Hamiltonian contains three terms corresponding to the electronic subsystem, vibrational subsystem, and excitation transfer. The approximations below are developed for the Jaynes-Cummings Hamiltonian, but can be equally obtained for a nonadiabatic Hamiltonian with the momentum dependent coupling $g\left(a^{+}-a\right)\left(S_{+}+S_{-}\right)$rather than the position dependent coupling $g\left(a^{+}+a\right)\left(S_{+}+S_{-}\right)$. A further simplification of the 
Jaynes-Cummings Hamiltonian is obtained by invoking the rotating wave or resonance approximation that keeps only those coupling terms that preserve the total number of quanta in the combined electron-vibrational system.

$$
H_{\mathrm{JCM}}=\omega\left(a^{+} a+1 / 2\right)+\Omega S_{+} S_{-}+g\left(a^{+} S_{-}+a S_{+}\right) .
$$

The dynamics that follow from this final form of the Hamiltonian are investigated below.

The expectation value of the $S_{\mathrm{z}}$ operator is the main focus of the present study. This expectation value gives populations of the electronic states of the dimer and, more generally, is related to the value of a reaction coordinate in exciton, electron, proton transfer and other chemical processes. In the Heisenberg representation of quantum mechanics, the expectation value of $S_{z}$ evolves in time through the time-dependence of the operator. The wave function remains fixed and is specified by the initial conditions. The Heisenberg equation of motion (EOM) for the operator $S_{\mathrm{z}}$ is given by its commutator with the Hamiltonian (14). The time-derivative of $S_{z}$ depends on other time-dependent operators, whose EOMs are also obtained by commutation with (14). This leads to an infinite hierarchy of EOMs. Noting that $S_{\mathrm{z}}=1 / 2\left(S_{+} S_{-}-S_{-} S_{+}\right)$, the infinite hierarchy can be written in a compact form using only three additional operators

$$
\begin{aligned}
& \hat{\alpha}=a^{+} S_{-}+a S_{+}, \\
& \hat{\beta}=a^{+} S_{-}-a S_{+}, \\
& \hat{\gamma}=a^{+} a+S_{+} S_{-}+\frac{1}{2},
\end{aligned}
$$

that involve the non-interacting Hamiltonian $\gamma$, the interaction part of the Hamiltonian $\alpha$, and the auxiliary operator $\beta$. The following hierarchy of Heisenberg EOMs is obtained starting with the expectation value of the population inversion operator $S_{\mathrm{z}}$

$$
\begin{aligned}
i \frac{d}{d t}\left\langle\alpha \gamma^{n}\right\rangle & =-\delta\left\langle\beta \gamma^{n}\right\rangle, \\
i \frac{d}{d t}\left\langle\beta \gamma^{n}\right\rangle & =-\delta\left\langle\alpha \gamma^{n}\right\rangle+\underbrace{g\left\langle S_{z} \gamma^{n+1}\right\rangle}_{\text {needs closure }}, \\
i \frac{d}{d t}\left\langle S_{z} \gamma^{n}\right\rangle & =g\left\langle\beta \gamma^{n}\right\rangle
\end{aligned}
$$

with $n=0,1,2, \ldots$. The detuning $\delta=\Omega-\omega$ denotes the difference between the electronic energy gap $\Omega$ and the vibrational frequency $\omega$. The Heisenberg EOMs for the operators are 
arranged in blocks of $n$-th order with three equations per block. The evolution of the lower order block depends on the evolution of the higher order block through the coupling term labeled in (16) by underbrace.

Useful approximations to the exact solution of the infinite chain of equations (16) can be obtained by limiting the number of equations to a few lower order blocks and decomposing the higher order coupling term into a product of lower order terms in the spirit of the quantized Hamiltonian dynamics (QHD) approach originally introduced for the classicallike expectation values of the position and momentum operators ${ }^{30,40}$. Decomposition of the higher order expectation values into products of the lower order ones terminates the infinite hierarchy (16) within a finite number of equations. With no a priori knowledge of the relationship between the expectation values of the higher and lower order operators, the contributions of all possible products of the lower order operators that represent the higher order operator are taken with equal weights ${ }^{40,43}$. The closure applied to decompose higher order moments of the position and momentum operators into products of the first order expectation values gives classical Hamiltonian mechanics. Decomposition of the higher order moments of position and momentum into products of the first and second moments leads to the well known Gaussian approximation ${ }^{41}$. Generally, the QHD procedure allows one to obtain simple classical-like EOMs for essentially quantum mechanical characteristics, such as zero-point energy, tunneling, quantum state populations, state-to-state correlations.

In present, the hierarchy (16) is terminated at $n=1$ requiring decomposition of the $S_{z} \gamma^{2}$ term. Using the general expression for the decomposition of a triple product ${ }^{40}$

$$
\begin{aligned}
\langle\hat{A} \hat{B} \hat{C}\rangle \simeq & \langle\hat{A}\rangle\langle\hat{B} \hat{C}\rangle+\langle\hat{B}\rangle\langle\hat{A} \hat{C}\rangle \\
& +\langle\hat{C}\rangle\langle\hat{A} \hat{B}\rangle-2\langle\hat{A}\rangle\langle\hat{B}\rangle\langle\hat{C}\rangle
\end{aligned}
$$

this term is decomposed into

$$
\left\langle S_{\mathrm{z}} \gamma^{2}\right\rangle \simeq 2\left\langle S_{\mathrm{z}} \gamma\right\rangle\langle\gamma\rangle+\left\langle S_{\mathrm{z}}\right\rangle\left\langle\gamma^{2}\right\rangle-2\left\langle S_{\mathrm{z}}\right\rangle\langle\gamma\rangle^{2},
$$


yielding a closed system of six differential equations

$$
i \frac{d}{d t}\left(\begin{array}{c}
\langle\alpha\rangle \\
\langle\beta\rangle \\
\left\langle S_{\mathrm{z}}\right\rangle \\
\langle\alpha \gamma\rangle \\
\langle\beta \gamma\rangle \\
\left\langle S_{\mathrm{z}} \gamma\right\rangle
\end{array}\right)=\left(\begin{array}{cccccc}
0 & -\delta & 0 & 0 & 0 & 0 \\
-\delta & 0 & 0 & 0 & 0 & g \\
0 & g & 0 & 0 & 0 & 0 \\
0 & 0 & 0 & 0 & -\delta & 0 \\
0 & 0 & \left\langle\gamma^{2}\right\rangle-2\langle\gamma\rangle^{2} & -\delta & 0 & 2\langle\gamma\rangle \\
0 & 0 & 0 & 0 & g & 0
\end{array}\right)\left(\begin{array}{c}
\langle\alpha\rangle \\
\langle\beta\rangle \\
\left\langle S_{\mathrm{z}}\right\rangle \\
\langle\alpha \gamma\rangle \\
\langle\beta \gamma\rangle \\
\left\langle S_{\mathrm{z}} \gamma\right\rangle
\end{array}\right) .
$$

Usually, closures produce systems of non-linear differential equations, such as the non-linear classical Hamiltonian EOMs obtained from the Heisenberg quantum EOMs by decomposition of the expectation values of higher order moments of position and momentum operators into products of the first order moments ${ }^{30,40}$. The special properties of the Jaynes-Cummings Hamiltonian in the rotating wave approximation, $[\hat{\gamma}, H]=0$ in particular, make Eqs. (19) into a system of linear differential equations. The operators $\gamma$ and $\gamma^{2}$ appearing in the closure (18) are integrals of motion of the Hamiltonian (14). The quadratic $\langle\gamma\rangle^{2}$ term in (19) is a constant specified by the initial conditions. Equations (19) give a very simple approximation to the dynamics of the electron-phonon system.

\section{ANALYSIS OF DYNAMICS}

The closed system of Heisenberg equations (19) can be solved analytically for the expectation value of the population inversion $\left\langle S_{z}\right\rangle$. The time-evolution of $\left\langle S_{z}\right\rangle$ is given by a superposition of two cosine functions,

$$
\begin{aligned}
\left\langle S_{\mathrm{z}}\right\rangle(t)=\left\langle S_{\mathrm{z}}\right\rangle(t=0) & +\frac{g^{2} \sqrt{\gamma_{0}}\left[\sqrt{\gamma_{0}}+1\right]}{2 \omega_{1}^{2}}\left(1-\cos \omega_{1} t\right) \\
& +\frac{g^{2} \sqrt{\gamma_{0}}\left[\sqrt{\gamma_{0}}-1\right]}{2 \omega_{2}^{2}}\left(1-\cos \omega_{2} t\right),
\end{aligned}
$$

whose frequencies are defined by

$$
\begin{aligned}
& \omega_{1}^{2}=\delta^{2}+2 g^{2}\left(\gamma_{0}+\sqrt{\gamma_{0}}\right) \\
& \omega_{2}^{2}=\delta^{2}+2 g^{2}\left(\gamma_{0}-\sqrt{\gamma_{0}}\right), \\
& \gamma_{0}=\langle\gamma\rangle(t=0) .
\end{aligned}
$$

The difference in the squares of the frequencies is given by the product of the square of the

electron-phonon coupling constant $g^{2}$ and the square root of the mean number of quanta 
in the system $\sqrt{\gamma_{0}}$. As should be expected, a perfect oscillation in the electronic state population is observed with zero electron-phonon coupling. As the strength of the electronphonon coupling grows, the difference between the two frequencies increases, leading to the dephasing of the electronic state population and a corresponding decrease in the oscillation amplitude, Fig. 2.The oscillation of population inversion will proceed more rapidly if the vibrational mode contains more energy. Vibrational motion of a large amplitude accelerates transfer of electronic population. The time evolution of the coupled electron-phonon system is illustrated in Fig. 2for the initial conditions

$$
\begin{aligned}
\left.\left\langle S_{\mathrm{z}}\right\rangle\right|_{t=0} & =-\frac{1}{2} \\
\left.\langle\gamma\rangle\right|_{t=0} & =\left\langle a^{+} a>,\right. \\
\left.\langle\alpha\rangle\right|_{t=0} & =0,\left.\langle\beta\rangle\right|_{t=0}=0 .
\end{aligned}
$$

The vibrational mode is prepared in a quasiclassical coherent state described in the coordinate representation by a Gaussian displaced from the vibrational equilibrium. All electronic population is localized in one state. There is no correlation between the electronic and vibrational subsystems at the initial time. The evolution predicted by Eq. (20) is compared with the exact solution ${ }^{49}$. The exact and approximate solutions coincide with good precision until $2 \pi g t<1$ holds $^{53}$, corresponding to $t<6.366$ and $t<0.6366$ in Figs. 2(a) and 2(b), respectively. The approximate solution is analytic, in a simple closed form, Eq. 20 compared to the exact solution involving an infinite series summation ${ }^{49}$. Note that within the time interval determined by $2 \pi g t<1$, the dynamics of inversion has relaxational character and usually fits the expression $\frac{1}{2} \cos \left(2 \sqrt{\gamma_{0}} g t\right) \exp \left(-g^{2} t^{2}\right)^{53}$.

The sum of the cosine functions in Eq. (20) forms beats. The fast oscillation of the population inversion in Fig. 2 gives excitation transfer between the electronic states. The envelope of the beats describes relaxation in the oscillation of the electronic population transfer due to the vibration induced dephasing. The inversion $t_{+}^{-1}$ and relaxation $t_{-}^{-1}$ rates

$$
\begin{aligned}
& t_{+}^{-1}=\frac{\omega_{1}+\omega_{2}}{2}, \\
& t_{-}^{-1}=\frac{\omega_{1}-\omega_{2}}{2} .
\end{aligned}
$$

depend on the number of phonons. The sum and difference of the frequencies, determine the beats of the electronic population in Eq. (20). The analytic dependence of the rates of in- 
version (faster component) and relaxation (slower component) on the vibrational amplitude $\left\langle\gamma_{0}\right\rangle$ and electron-vibrational detuning $\delta=\omega-\Omega$ is illustrated in Fig. 3 .

The dependence of $t_{ \pm}^{-1}$ on $\delta$ and $\left\langle\gamma_{0}\right\rangle$ becomes clear from the following series expansion: For $\delta \ll 1$ and $\left\langle\gamma_{0}\right\rangle \gg 1$ the frequencies $t_{ \pm}$of the population inversion oscillation and relaxation can be expanded in powers of $\delta$ and $1 / \sqrt{\left\langle\gamma_{0}\right\rangle}$

$$
\begin{aligned}
& t_{+}^{-1} \simeq g \sqrt{2\left\langle\gamma_{0}\right\rangle}-\frac{g}{2 \sqrt{2\left\langle\gamma_{0}\right\rangle}}+\frac{\delta^{2}}{2 g \sqrt{2\left\langle\gamma_{0}\right\rangle}}+\ldots, \\
& t_{-}^{-1} \simeq \frac{g}{\sqrt{2}}\left(1+\frac{1}{8\left\langle\gamma_{0}\right\rangle}\right)-\frac{1}{4 \sqrt{2}} \frac{\delta^{2}}{g\left\langle\gamma_{0}\right\rangle}+\ldots
\end{aligned}
$$

The inversion rate $t_{+}^{-1}$ has minimum at $\delta=0$, the relaxation rate $t_{-}^{-1}$ has maximum at $\delta=0$. A larger detuning leads to faster oscillations of population and a slower relaxation.

The dependence of $t_{ \pm}$on the amount of vibrational energy is considered in the $\delta=0$ limit, which has been explored in Fig. 2. Expansion of the sum and difference frequencies up to the third order in $1 / \sqrt{\left\langle\gamma_{0}\right\rangle}$ with $\delta=0$ takes the form:

$$
\begin{aligned}
& t_{+}^{-1} \simeq g \sqrt{2\left\langle\gamma_{0}\right\rangle}-\frac{g}{2 \sqrt{2\left\langle\gamma_{0}\right\rangle}}+\ldots, \\
& t_{-}^{-1} \simeq \frac{g}{\sqrt{2}}\left(1+\frac{1}{8\left\langle\gamma_{0}\right\rangle}\right)+\ldots
\end{aligned}
$$

The frequency of population inversion grows with system energy $\left\langle\gamma_{0}\right\rangle=\bar{\alpha}^{*} \bar{\alpha}$, already in the zeroth order of expansion. The relaxation frequency decreases as inverse of system energy, Fig. 3. Considering the vibrational mode as a quasi heat bath with respect to the two-level system, it is intuitively expected that hoter bath yields quicker relaxation. The decrease of the relaxation rate with increasing phonon energy occurs since the oscillator is not in a thermal state, but approaches at large $\left\langle\gamma_{0}\right\rangle$ the classical regime. A classical oscillator coupled to a two state electronic system yields oscillation in the electronic population without relaxation. For small $\left\langle\gamma_{0}\right\rangle \leq 1$ the oscillation and relaxation frequences coincide. For large $\left\langle\gamma_{0}\right\rangle>1$, as indicated by expansion (25) the oscillation frequency grows while the relaxation frequency decreases. The frequencies display branching with energy growth, Fig. 3.

The approximate solution Eq. (20) not only gives the first few oscillations of the electronic population corresponding to several forward and backward population transfer reactions, but also well reproduces the overall dephasing envelope that is associated in the thermodynamic limit of many vibrational modes with relaxation to equilibrium. It is quite remarkable that this quasi-equilibration is observed transiently already with a single vibrational mode! 
It is instructive to look further into the dynamics of the population inversion $\left\langle S_{z}\right\rangle$. The dynamics of this observable is conjugate to the dynamics of the expectation value of the correlation operator $\langle\beta\rangle$, defined in Eqs. (15). As shown by the exact solution in Fig. 4, after a few oscillations the population inversion stalls at zero indicating that the electronic population is equally delocalized between the two states. This situation is referred to as "collapse" 54 . After a while, the oscillations reappear, Fig. 4, producing a "revival" ${ }^{55}$. It is important to understand in more detail this type of dynamics, in particular, the fate of the energy and phase dynamics during the "silent period", after the collapse and before the revival. The electronic contribution to the total energy is stored in an unusual form during the silent period. The electronic population is distributed equally between the two states. This situation is equivalent to the high temperature limit of the populations of a two-level system coupled to a thermostat. Here the nontrivial dynamics appear due to the entanglement of the electronic and vibrational degrees of freedom.

It is intriguing what happens to the phase of the oscillation during the silent period. The phase is preserved in a hidden manner to reappear later when the silent period is over, Fig. 4. It may be expected that a simple operator or a combination of several operators exist, whose expectation value maintains the oscillation, while the oscillation of the population inversion stalls. The dispersion

$$
\sigma_{A}=\left\langle(A-\langle A\rangle)^{2}\right\rangle .
$$

of the vibrational coordinate $(A=q)$ is the desired expectation value. The vibrational dispersion starts at the minimal value at time zero, grows with time and maintains the oscillation during the silent period, Fig. 5.

The origin of the oscillation in the dispersion of the vibrational coordinate is illustrated by the evolution of the vibrational wavepacket in Fig. 6 that shows wavepacket snapshots at several times. Initially, the wavepacket is Gaussian. As time evolves, the electronvibrational coupling splits the vibrational wavepacket into two branches correlated with the two electronic states. The dispersion of the vibrational coordinate represents the width of the overall wavepacket. The width oscillates as the two wavepacket branches evolve nearly independently as determined by their own electronic states. The width of the overall wavepacket is determined by the separation between the branches. The width is maximal when the two wavepackets are far apart and minimal when the two wavepackets penetrate 
through each other. After the silent period when the population inversion resumes its oscillation, the wavepacket width oscillation decreases in amplitude, Fig. 6.

\section{ANALYSIS OF UNCERTAINTY}

Dephasing of the electronic subsystem due to coupling to vibrations is ubiquitous in photochemistry. The silent period when the dynamics of the electronic population in the Jaynes-Cummings model temporarily stops due to the vibrationally induced dephasing is investigated further. Consider the uncertainties of the observables describing the vibrational and electronic modes. The vibrational wavepacket with two peaks that exist during the silent period, Fig. 6, has a large uncertainty. As shown before, the phase of the electronic subsystem is preserved in a hidden form by the uncertainty of the vibrational coordinate.

The uncertainty relationship for dispersions $\sigma_{A}, \sigma_{B}$, Eq. (26), of Hermitian operators $A$ and $B$ is commonly written in a product form as ${ }^{56}$

$$
\sigma_{A} \cdot \sigma_{B} \geq\left|\frac{1}{4}\langle[A, B]\rangle^{2}\right|
$$

An alternative relationship between the dispersions in a sum form can be obtained based

on the positivity of the norm of operator $F=A-\langle A\rangle+i(B-\langle B\rangle),\left\langle F^{*} F\right\rangle \geq 0 \rightarrow$

$$
\begin{array}{r}
\left\langle(A-\langle A\rangle)^{2}+(B-\langle B\rangle)^{2}+i[A-\langle A\rangle, B-\langle B\rangle]\right\rangle \geq 0, \text { and } \\
\sigma_{A}+\sigma_{B}+i\langle[A, B]\rangle \geq 0 .
\end{array}
$$

The sum $\sigma_{A}+\sigma_{B}+i\langle[A, B]\rangle$ gives a good second-order combination of operators, whose expectation value can be included into the chain of Heisenberg EOMs. In application to the vibrational subsystem, the sum uncertainty relationship (28) means that the sum of dispersions for the vibrational position and momentum

$$
\begin{aligned}
& A=a^{+}+a=q \sqrt{2 m \omega / \hbar} \\
& B=i\left(a^{+}-a\right)=p \sqrt{2 /(\hbar m \omega)}
\end{aligned}
$$

must be larger than zero

$$
\left\langle a^{+} a\right\rangle-\left\langle a^{+}\right\rangle\langle a\rangle \geq 0
$$


Consider the dispersion of coordinate in more detail. It can be written in terms of the raising and lowering operators in the form

$$
\begin{aligned}
\sigma_{q} & =\left\langle(q-\langle q\rangle)^{2}\right\rangle \\
& =\frac{\hbar}{2 m \omega}\left\langle\left[a^{+}+a-\left\langle a^{+}+a\right\rangle\right]^{2}\right\rangle \\
& =\frac{\hbar}{2 m \omega}\{\underbrace{\left\langle a a^{+}\right\rangle-\langle a\rangle\left\langle a^{+}\right\rangle+\left\langle a^{+} a\right\rangle-\left\langle a^{+}\right\rangle\langle a\rangle}_{\text {cross term }} \underbrace{\left\langle\left\langle a^{+}\right)^{2}\right\rangle-\left\langle a^{+}\right\rangle^{2}+\left\langle a^{2}\right\rangle-\langle a\rangle^{2}}_{\text {ladder term }}\} .
\end{aligned}
$$

The coordinate dispersion includes two kinds of terms, the ladder dispersion terms involving operator squares that increase or decrease the number of quanta by two, and the cross terms including products of conjugate ladder operators that preserve the number of quanta. The mean value of the ladder term oscillates quickly and gives fringes in Fig. 5. The cross term maintains the rotating wave approximation preserving the number of quanta, and evolves slowly. Similar decomposition applies to the dispersion of momentum

$$
\begin{aligned}
\sigma_{p} & =\frac{m \omega \hbar}{2}\left\langle\left[a^{+}-a-\left\langle a^{+}-a\right\rangle\right]^{2}\right\rangle \\
& =\frac{m \omega \hbar}{2}\{\underbrace{\left\langle a a^{+}\right\rangle-\langle a\rangle\left\langle a^{+}\right\rangle+\left\langle a^{+} a\right\rangle-\left\langle a^{+}\right\rangle\langle a\rangle}_{\text {cross term }} \underbrace{\left.-\left[\left\langle a^{+}\right)^{2}\right\rangle-\left\langle a^{+}\right\rangle^{2}+\left\langle a^{2}\right\rangle-\langle a\rangle^{2}\right]}_{\text {ladder term }}\} .
\end{aligned}
$$

The difference from the position operator dispersion is only in the negative sign by the ladder term. The quickly oscillating ladder terms cancel out in the sum of the coordinate and momentum dispersions, leaving only the cross terms

$$
\frac{1}{4}\left(\sigma_{A}+\sigma_{B}\right)=\frac{m \omega}{2 \hbar} \sigma_{q}+\frac{1}{2 m \omega \hbar} \sigma_{p}=\{\underbrace{\left\langle a^{+} a\right\rangle+1 / 2}_{\text {total energy }}-\underbrace{\left\langle a^{+}\right\rangle\langle a\rangle}_{\text {semiclassical }}\}=E_{\text {quant }}^{\mathrm{vib}} .
$$

The slowly oscillating sum of dispersions (33) is nothing but a purely quantum contribution to the vibrational energy defined as the difference between the total vibrational energy

$$
E_{\text {total }}^{\mathrm{vib}}=\frac{1}{2 m}\left\langle p^{2}\right\rangle+\frac{m \omega^{2}}{2}\left\langle q^{2}\right\rangle=\hbar \omega\left\langle a^{+} a+1 / 2\right\rangle,
$$

and the semiclassical contribution to the total energy

$$
E_{\mathrm{semi}-\mathrm{cl}}^{\mathrm{vib}}=\frac{1}{2 m}\langle p\rangle^{2}+\frac{m \omega^{2}}{2}\langle q\rangle^{2}=\hbar \omega\left\langle a^{+}\right\rangle\langle a\rangle,
$$

formed of the first order classical-like averages $\langle q\rangle$ and $\langle p\rangle$. The purely quantum contribution to the vibrational energy provides the envelope of the coordinate dispersion in Fig. 5. Substitution of the commutation relation $[p, q]=i \hbar \rightarrow[A, B]=[q \sqrt{2 m \omega / \hbar}, p \sqrt{2 / m \omega \hbar}]=2 i$ 
and the sum of dispersions (33) into the general formula (28) gives the uncertainty relationship (30) for the vibrational dispersions.

Next, consider the sum uncertainty relationship, Eq. (28), for the electronic, generally fermionic subsystem with

$$
\begin{aligned}
A & =\frac{1}{2}\left(S_{+}+S_{-}\right)=S_{\mathrm{x}}, \\
B & =\frac{i}{2}\left(S_{+}-S_{-}\right)=S_{\mathrm{y}} .
\end{aligned}
$$

The inequality (28) gives

$$
\left\langle S_{+} S_{-}\right\rangle-\left\langle S_{+}\right\rangle\left\langle S_{-}\right\rangle \geq 0
$$

Similarly to the vibrational subsystem, the dispersions of the electronic $S_{\mathrm{x}}$ and $S_{\mathrm{y}}$ operators can be decomposed into the cross and ladder terms. The dispersion $\sigma_{S_{\mathrm{x}}}$ reads

$$
\begin{aligned}
\sigma_{S_{\mathrm{x}}} & =\frac{1}{4}\left\langle\left[S_{+}+S_{-}-\left\langle S_{+}+S_{-}\right\rangle\right]^{2}\right\rangle \\
& =\frac{1}{4}\{\underbrace{\left\langle S_{+} S_{-}\right\rangle-\left\langle S_{+}\right\rangle\left\langle S_{-}\right\rangle+\left\langle S_{-} S_{+}\right\rangle-\left\langle S_{-}\right\rangle\left\langle S_{+}\right\rangle}_{\text {cross term }} \underbrace{+\left\langle S_{+}^{2}\right\rangle-\left\langle S_{+}\right\rangle^{2}+\left\langle S_{-}^{2}\right\rangle-\left\langle S_{-}\right\rangle^{2}}_{\text {ladder term }}\} .
\end{aligned}
$$

Since the second order averages in the last equation add to a constant, $\left\langle S_{+} S_{-}+S_{-} S_{+}\right\rangle=1$ by completeness, and $\left\langle S_{+}\right\rangle^{2}=\left\langle S_{-}\right\rangle^{2}=0$ due to finite dimensionality of the electronic basis, the electronic dispersion involves only the first order averages

$$
\sigma_{S_{\mathrm{x}}}=1 / 4(\underbrace{1-2\left\langle S_{+}\right\rangle\left\langle S_{-}\right\rangle}_{\text {cross }} \underbrace{-\left\langle S_{+}\right\rangle^{2}-\left\langle S_{-}\right\rangle^{2}}_{\text {ladder }}) .
$$

The ladder terms oscillate quickly and are responsible for the fringes in Fig. 7. Similarly, the dispersion of $S_{\mathrm{y}}$ can be decomposed into the cross and ladder terms

$$
\sigma_{S_{\mathrm{y}}}=1 / 4(\underbrace{1-2\left\langle S_{+}\right\rangle\left\langle S_{-}\right\rangle}_{\text {cross }} \underbrace{+\left\langle S_{+}\right\rangle^{2}+\left\langle S_{-}\right\rangle^{2}}_{\text {ladder }}) .
$$

The quickly oscillating ladder terms cancel out in the sum of the two dispersions

$$
\sigma_{S_{\mathrm{x}}}+\sigma_{S_{\mathrm{y}}}=\frac{1}{2}-\left\langle S_{+}\right\rangle\left\langle S_{-}\right\rangle
$$

The sum of the electronic dispersions up to a constant and a sign is nothing but the quasiclassical contribution

$$
E_{\text {quasicl }}^{\mathrm{el}}=\hbar \Omega\left\langle S_{+}\right\rangle\left\langle S_{-}\right\rangle
$$


to the total electronic energy

$$
E_{\text {total }}^{\mathrm{el}}=\hbar \Omega\left\langle S_{+} S_{-}\right\rangle
$$

Note that the corresponding sum of vibrational dispersions gives the quantum contribution to vibrational energy, Eq. (33). The difference between $E_{\text {quasicl }}^{\mathrm{el}}$ and $E_{\text {total }}^{\mathrm{el}}$ then is the purely quantum contribution to the electronic energy

$$
E_{\text {quantum }}^{\mathrm{el}}=E_{\text {total }}^{\mathrm{el}}-E_{\text {quasicl }}^{\mathrm{el}}=\hbar \Omega\left(\left\langle S_{+} S_{-}\right\rangle-\left\langle S_{+}\right\rangle\left\langle S_{-}\right\rangle\right)
$$

Substitution of the commutator $\left[S_{\mathrm{x}}, S_{\mathrm{y}}\right]=-i S_{\mathrm{z}}$ and sum of electronic dispersions Eq. (41) into the general formula (28) gives the relation (37) between the electronic dispersions in an equivalent form

$$
\sigma_{S_{\mathrm{x}}}+\sigma_{S_{\mathrm{y}}}+\left\langle S_{\mathrm{z}}\right\rangle \geq 0
$$

with $S_{\mathrm{z}}=S_{+} S_{-}-1 / 2$ and Eq. (41). Eqs. (37) and (45) imply that the purely quantum part of the electronic energy is always greater than zero, $E_{\text {quant }}^{\mathrm{el}} \geq 0$. Over time the electronic energy flows between its quasiclassical and quantum parts, as shown in Fig. 7. The quasiclassical contribution to the electronic energy provides an envelope for the electronic dispersion.

The electronic and vibrational dispersions show interesting similarities that are summarized in Table I. The fringes in the electronic dispersion, Fig. 7, appear at the same phase and frequency as the fringes in the vibrational dispersion, Fig. 5. The difference is in the sign of the deviation of the electronic and vibrational dispersions from their bounds, cf. the signs in inequalities (30) and (37). The fringes in the vibrational dispersion are directed upward, while the fringes in the electronic dispersion are directed downward. The electronic dispersion is bounded from above in contrast to the vibrational dispersion, which is bounded from below.

Figure 8 displays the sums of dispersions for the vibrational and subsystems in comparison to the real valued electron-vibrational correlation

$$
i\left\langle\beta_{r}\right\rangle=\left\{\langle\beta\rangle-\langle a\rangle\left\langle S_{+}\right\rangle-\left\langle a^{+}\right\rangle\left\langle S_{+}\right\rangle\right\}
$$

directly related to the auxiliary operator $\hat{\beta}$, Eq. (15). The correlation oscillates with the same low frequency as the electronic and vibrational dispersions. Rigorously, the combination of operators Eq. (46) can naturally appear as a sum of dispersions $\sigma_{A}+\sigma_{B}$ for cross-operators, 
composed of both fermionic and bosonic parts $A=S_{+}+a, B=i\left(S_{+}-a\right)$ and their conjugates. For the initial condition with no correlation between the fermionic and bosonic modes, and zero horizontal spin projections $\left.\left\langle S_{+}\right\rangle\right|_{t=0}=0$, the expectation value of Eq. (46) starts at zero and oscillates around zero. Up to zero point value and scaling constants, the dispersions of electronic subsystem and the correlation Eq. (46) behave as sine and cosine functions.

When the population inversion stalls, Fig. 4, the electron-phonon system is in a state with large uncertainty. For both electronic and vibrational subsystems, states with larger uncertainties provide larger purely quantum energy contributions. As shown in Fig. 5, the dispersion associated with the vibrational subsystem increases, in accord with the uncertainty relationship (30). In contrast to the vibrational dispersion, the dispersion associated with the electronic subsystem decreases as seen in Fig. 7, in accordance with the uncertainty relationship (37). The electronic subsystem approaches the state of minimal uncertainty when inversion oscillation stops.

The unusual behavior of the dispersion associated with the electronic subsystem can be understood with the concept of pure and mixed states. An isolated electronic system is characterized by either a normalized wave function $|\psi\rangle=c_{0}|0\rangle+c_{1}|1\rangle$, or a density matrix, or quantum mechanically averaged values of electronic operators. In an entangled electronphonon system the electronic part is a statistical average over different realizations of the vibrational subsystem. The statistical mixture of states cannot be represented through a wave function, and is characterized by a reduced density matrix or both statistically and quantum mechanically averaged values of electronic operators.

The density matrix of an isolated electronic system is determined by the products of the wave function coefficients $\rho_{\mathrm{el}}=|\psi\rangle\left\langle\psi\left|=c_{1}^{*} c_{1}\right| 1\right\rangle\left\langle 1\left|+c_{1}^{*} c_{0}\right| 1\right\rangle\left\langle 0\left|+c_{0}^{*} c_{1}\right| 0\right\rangle\left\langle 1\left|+c_{0}^{*} c_{0}\right| 0\right\rangle\langle 0|$. The reduced density matrix of the electronic subsystem of the electron-phonon system can be specified by expectation values of electronic operators averaged over the state of the whole electron-phonon system $\rho_{\mathrm{el}}=\left\langle S_{+} S_{-}\right\rangle|1\rangle\left\langle 1\left|+\left\langle S_{+}\right\rangle\right| 1\right\rangle\left\langle 0\left|+\left\langle S_{-}\right\rangle\right| 0\right\rangle\left\langle 1\left|+\left\langle S_{-} S_{+}\right\rangle\right| 0\right\rangle\langle 0|$. The trace of the squared density matrix of an isolated electronic system is a complete square and always equals unity $\operatorname{Tr} \rho_{\mathrm{el}}^{2} \equiv\left(c_{1}^{*} c_{1}+c_{0}^{*} c_{0}\right)^{2}=1$, due to normalization of the wave function. The trace of the reduced electronic density matrix squared, often referred to as fidelity, takes 
the form

$$
\operatorname{Tr} \rho_{\mathrm{el}}^{2}=1+2\left\langle S_{+}\right\rangle\left\langle S_{-}\right\rangle-\left\langle S_{+} S_{-}\right\rangle\left\langle S_{-} S_{+}\right\rangle \leq 1
$$

and is less or equal one. The last term is nonnegative, because it is a product of nonnegative populations $\left\langle S_{ \pm} S_{\mp}\right\rangle \geq 0$. The second term is zero if the averaging over the phonon state gives no correlation between $|0\rangle$ and $|1\rangle$. $\operatorname{Tr} \rho_{\mathrm{el}}^{2}=1$ means that the electronic subsystem exists in a pure state. $\operatorname{Tr} \rho_{\mathrm{el}}^{2}<1$ implies that the electronic subsystem is in a mixed state.

The distinction between pure and mixed states is best illustrated with the Bloch vector

$$
\vec{R} \equiv\left(\left\langle S_{\mathrm{x}}\right\rangle,\left\langle S_{\mathrm{y}}\right\rangle,\left\langle S_{\mathrm{z}}\right\rangle\right)
$$

The square of the Bloch vector $R^{2}$ is related to $\operatorname{Tr} \rho_{\text {el }}^{2}$ by a linear transformation given in Table II. The Bloch vector (48) connects the origin with the point in a three-dimensional space specified by the expectation values of the projections of the spin- $1 / 2$ operator onto $\mathrm{x}$, y, z axes.

$$
R^{2}=\left\langle S_{\mathrm{x}}\right\rangle^{2}+\left\langle S_{\mathrm{y}}\right\rangle^{2}+\left\langle S_{\mathrm{z}}\right\rangle^{2} \leq 1 / 4
$$

The square of the Bloch vector can be decomposed $R^{2}=\left\langle S_{\mathrm{z}}\right\rangle^{2}+R_{\mathrm{xy}}^{2}$ into the vertical $\left\langle S_{\mathrm{z}}\right\rangle$ and horizontal components

$$
R_{\mathrm{xy}}^{2}=\left\langle S_{\mathrm{x}}\right\rangle^{2}+\left\langle S_{\mathrm{y}}\right\rangle^{2}=\left\langle S_{-}\right\rangle\left\langle S_{+}\right\rangle
$$

The $\mathrm{x}, \mathrm{y}$, and $\mathrm{z}$ components of the Bloch vector of an isolated electronic system are $\left(c_{1}^{*} c_{0}+c_{0}^{*} c_{1}\right) / 2, \quad\left(c_{1}^{*} c_{0}-c_{0}^{*} c_{1}\right) / 2 i, \quad\left(c_{1}^{*} c_{1}-c_{0}^{*} c_{0}\right) / 2 \quad$ yielding $R^{2}=$ $\frac{1}{4}\left[\left(c_{1}^{*} c_{1}\right)^{2}+\left(c_{0}^{*} c_{0}\right)^{2}+2 c_{1}^{*} c_{1} c_{0}^{*} c_{0}\right]=1 / 4$. The squared length of the Bloch vector of the electronic subsystem of a coupled electron-phonon system is

$$
R^{2}=1 / 4+\left\langle S_{+}\right\rangle\left\langle S_{-}\right\rangle-\left\langle S_{+} S_{-}\right\rangle\left\langle S_{-} S_{+}\right\rangle \leq 1 / 4
$$

Here, the last term is always nonnegative, and the second term is zero if the correlation between 0 and 1 is dephased by averaging over the vibrational states. $R^{2}$ decreases to zero when the electronic subsystem is equally mixed between the two states, $\left\langle S_{+} S_{-}\right\rangle=$ $\left\langle S_{-} S_{+}\right\rangle=1 / 2$, the last term compensating the $1 / 4$ constant. The relationships between the square of the Bloch vector and the trace of the squared density matrix are summarized in Table II. As shown in Fig. 8(c) for electronic subsystems with equal populations of both 
electronic states $\left\langle S_{z}\right\rangle=0,\left\langle S_{+} S_{-}\right\rangle=\left\langle S_{-} S_{+}\right\rangle=1 / 2$, the change of the squared Bloch vector from 0 to $1 / 4$ corresponds to the change of fidelity from $1 / 2$ to 1 . Fidelity expressed in terms of the electronic dispersions shows that mixed electronic states correspond to larger values of the electronic dispersions. Pure electronic states yield minimal uncertainties of electronic operators, minimal values for the electronic dispersions and the maximal length of the Bloch vector $\mathrm{R}=1 / 2$. Alternatively, mixed electronic states yield larger electronic operator uncertainties, larger electronic dispersions and shorter Bloch vectors.

The end of the Bloch vector glides from the south pole of the Bloch sphere to the north pole then back to the south pole and so on. During this revolutions, shown in the lower panel of Fig. 9, the length of the Bloch vector decreases, as the electronic subsystem becomes a statistical mixture. The mixing is maximized at $\omega t \simeq 1.5-2.0$. For times $\omega t>2$ the Bloch vector becomes larger and the electronic subsystem returns closer to a pure state. The length of the Bloch vector never recovers its initial value during the studied time period. In the middle of the silent period the Bloch vector is almost at maximal length $R^{2} \simeq 1 / 2$. Its z-axis projection is zero $\left\langle S_{\mathrm{z}}\right\rangle=0$ in accordance with Fig. 4. Thus, during the silent period the Bloch vector revolves equatorially (nutates) around z-axis.

The analysis of fidelity shows that the electronic subsystem successively passes through the following sequence of states. Initially in a pure state, the electronic subsystem quickly becomes a mixed state with a small fidelity. This occurs within one-eighth of the revival period, defined by $\Omega_{\text {revival }} \simeq g /{\sqrt{\gamma_{0}}}^{55}$. Then, the fidelity rises and the electronic subsystem again closely approaches a pure state. The fidelity is maximized at exactly one half of the revival period, $t=\pi / \Omega_{\text {revival }}$.

During the silent period when the electronic population stalls, the oscillation shifts to the vibrational and electronic dispersions and $\left\langle S_{\mathrm{x}}\right\rangle,\left\langle S_{\mathrm{y}}\right\rangle$ expectation values that preserve the phase of the oscillation and are maximized exactly in the middle of the silent period. Generally, the electronic and vibrational dispersions and electronic coherences $\left\langle S_{\mathrm{x}}\right\rangle,\left\langle S_{\mathrm{y}}\right\rangle$ are maximized at half integer revival times $t_{\text {revival }}=2 \pi / \Omega_{\text {revival }}=2 \pi \sqrt{\gamma_{0}} / g$, while the electronic inversion $\left\langle S_{z}\right\rangle$ and vibrational classical-like $\langle p\rangle$ and $\langle q\rangle$ are maximized at integer revival times. 


\section{TIME SCALES}

The coupled electron-phonon dynamics in the molecular dimer forms a hierarchy of time scales. The electronic and vibrational coherences $\left\langle S_{\mathrm{x}}\right\rangle$ and $\langle q\rangle$, as well as electronic population $\left\langle S_{z}\right\rangle$ and energy $\left\langle a^{+} a\right\rangle$ oscillate with different frequencies that vary by order of magnitude or more. Each frequency component can be associated with a distinct physical process. The following is the hierarchy of the time scales in increasing order. The quickest time scale $t_{I}$ corresponds to the oscillations of the expectation values of the vibrational coordinate $\langle q\rangle$ and electronic coherence $\left\langle S_{\mathrm{x}}\right\rangle,\left\langle S_{\mathrm{y}}\right\rangle$. The second time scale $t_{I I}$ characterizes the inversion of the electronic population $\left\langle S_{z}\right\rangle$. The population inversion time depends on the initial displacement of the vibrational wavepacket. Larger displacements yield quicker population transfer. Relaxation and dephasing of the population inversion occurs on a slower time scale $t_{I I I}$. Over this time interval the electronic state approaches quasi-equilibrium with equal partitioning of the population between the two states. The slowest component $t_{I V}$ is associated with the silent period and determines the revival of the oscillation in the electronic state populations. The $t_{I V}$ time also determines the time scale of the relaxation of the expectation value of the vibrational coordinate $\langle q\rangle$ to its equilibrium position. The electron-vibrational system enters and leaves the state with the maximal uncertainty on the $t_{I V}$ time scale.

The time scales of the faster processes $I$ through $I I I$ can be estimated from the approximate analytic expressions (20)-(21) derived in this work. The slowest time scale $I V$ is estimated by Eberly et al. ${ }^{55}$ and is reproduced in our numerical simulations. The slowest time scale $I V$ describing the silent period and revivals of the oscillation in the electronic populations does not appear as a separate time scale in the analytic expressions (20)-(21) and coincides with the time scale $I I I$ of the relaxation and dephasing of the oscillation. The revivals in the electron-phonon system can take longer than the dephasing time and produce a silent period. According to Eberly et $a l .{ }^{55}, t_{I V}$ is determined by the difference in the Rabi flopping frequencies of the two neighboring and most populated levels of the oscillator. The following are the rates associated with the four time scales

$$
\begin{aligned}
t_{\mathrm{I}}^{-1} & \sim \omega_{\mathrm{vib}} \\
t_{\mathrm{II}}^{-1} & \sim g \sqrt{2\left\langle\gamma_{0}\right\rangle} \\
t_{\mathrm{III}}^{-1} & \sim g / \sqrt{2}
\end{aligned}
$$




$$
t_{\mathrm{IV}}^{-1} \sim \frac{g}{\sqrt{\left\langle\gamma_{0}\right\rangle}}
$$

Even for this simple two dimensional system isolated from environment, the time taking the system to return to its initial state, the Poincare recurrence time can be very long. The quasi-irreversibility feature appears due to discreteness of the oscillator states and determines the character of the long-time dynamics. After each revival period the state of the system is farther away from the initial state. For most real systems with large number of degrees of freedom even the first revival is barely accessible. According to Figures 2 and 5, after a long period of time the expectation values of the population inversion and vibrational coordinate lose the revivals and approach quasi-equilibrium. Figure 7 indicates that some electronic coherence may persist for a long time. Based on the above analysis it is reasonable to expect that after a sufficiently long time interval the system will be found in a state of large uncertainty with the first order dynamical averages performing small amplitude chaotic motions about the equilibrium values.

\section{DISCUSSION}

Few simple quantum models allow for analytic treatment: two interacting harmonic oscillators as well as two interacting two-level systems are exactly solvable. A harmonic oscillator interacting with a two-level system cannot be solved exactly by use of a finite number of variables. Known are the infinite series solutions of the eigenstate method and finite approximations by the QHD method, presented here. The infinite set of wave function coefficients in the equations of motion in the eigenstate solution is equivalent to the infinite chain of QHD equations.

The dynamics of a massive particle in a harmonic potential, undergoes the same evolution as a quantized mode of a massless electromagnetic wave. The relaxation of the population inversion is irreversible in general, so that the dynamics can be referred to as reversible

decoherence. An interesting issue is observed in Fig. 6(a) at $\omega t /(2 \pi)=9.5$. The dispersion of coordinate closely approaches its initial value. The states of the electronic and vibrational subsystems become correlated with time. This nontrivial behavior of the averages is associated with formation of entanglement.

Applications and extensions of this work cover three directions: theoretical, chemical, 
and spectroscopic. From the theoretical point of view the results can be improved by using higher order closure relations. The necessity to take into account zero-point energy effects and get a better description of the silent period suggests an extension of the calculation scheme by including equations of motion for new variables, in particular, the dispersions of products of nuclear and electronic operators. Variables which follow state-specific positions, electronic phase, and vibrational phase acquired by the dimer with time can be included into the calculation scheme for application to echo experiments. For instance, the statespecific positions are $\left\langle q\left(S_{\mathrm{z}} \pm 1 / 2\right)\right\rangle$. The relative electronic phase can be defined with $\left\langle S_{\mathrm{x}}\right\rangle$, $\left\langle S_{\mathrm{y}}\right\rangle$. The vibrational phase is specified by a variable, analogous to the $\gamma$ term in the Heller work on Gaussian wavepackets ${ }^{32}$. Some of these new variables and equations of motion appear naturally while describing the coupled electron-phonon dynamics with the FokkerPlanck equation of motion for the normally ordered multidimensional generating function, comprising both electronic and nuclear degrees of freedom ${ }^{57}$.

An important chemical application of the presented method of calculating the coupled electron-phonon dynamics is the description of energy and charge transfer in large molecular aggregates playing important biological and technological roles, e.g. in the light harvesting complex and molecular solar cells. The dynamics of electronic excitations is coupled in large systems to many inter- and intramolecular nuclear degrees of freedom as well as to vibrations of environment, whose dynamics are extremely difficult to follow on the quantum level. The QHD method used here follows the quantum properties using few expectation values to characterize each degree of freedom, and describes the exciton and electron transfer dynamics in a simple way. The QHD method is directly applicable to the quantitative description of transfer process in large chemical systems.

The method of this paper is also able to describe 3 pulse-, 4 pulse-, and correlated photon echo spectroscopic experiments with molecular systems by addition of new variables. In femtosecond echo experiments with ensembles of disordered dimers ${ }^{9,64}$, the evolution of phase for each dimer in the ensemble determines the re-phasing of the ensemble net transition dipole and, consequently, the photon echo signal. In particular, for the three-pulse stimulated photon echo technique, the phase acquired during the population period between pulses II and III determines the time delay for appearance of the stimulated echo. The echo signal determined by the relative phase between the ground and one of the excited electronic states, carries information about the population transfer between the optically active excited 
states. It is expected that oscillations in the third-order echo peak shift, modulated by the damped evolution of the excited states population $\left\langle S_{z}\right\rangle$, will be reproduced by the current method. In a four pulse femtosecond experiment, the fourth pulse serves for the heterodyning: The excited state wavepacket is projected onto the ground state wavepacket. The created interference population in the excited state affects the net fluorescence from this state. The time of the signal is determined by the acquired electronic phase and position of the exciton transfer wavepacket described by the $\left\langle q\left(S_{z} \pm 1 / 2\right)\right\rangle$ variable.

A novel correlated spectroscopic technique, based on a simultaneous irradiation of a sample by pulses of optical (electronic) and IR (vibrational) frequencies can yield new information about the dynamics of the electron-vibrational correlation c.f. $\langle\alpha\rangle$ and $\langle\beta\rangle$, calculated in this paper. We propose to excite and probe by correlated infrared and optical pulses. The scenario is analogous to the 2D IR spectroscopy used to get the coupling between the vibrational modes with close frequencies ${ }^{20}$. For the case of symmetric modes, the dynamics of coupling between optically active low frequency vibrational and high frequency electronic modes can be revealed by 2D Raman. For non-symmetric modes direct and independent excitation of the vibrational degree of freedom with an IR-pulse and the electronic degree of freedom with an optical pulse is required. The technical difficulty of this experimental method is establishing the phase relations between the IR and optical excitations. The experimental data measured by such technique can be modeled by the method developed in this paper.

\section{CONCLUSIONS}

Quantum dynamics of an electron-nuclear excitation in a model dimer has been analyzed in this work in the limit of small nuclear reorganization. The relaxation behavior in the model has been obtained by a reduced description that, by simplicity, is applicable to large systems. The approximate approach quantitatively reproduces the correct behavior of the model. It is also demonstrated that even in the absence of the heat bath the system approaches a mixed state corresponding to a quasi-thermal equilibrium, by destructive interference of wave function coefficients.

The influence of the vibrational mode onto the electronic population dynamics is analyzed by solving a chain of coupled differential equations for population inversion, electron- 
vibrational correlation, etc. The derived analytic result describes oscillation and relaxation of electronic population at short times. The approximate solution correctly represents the first several population inversions and the decay in the amplitude of the oscillation of the electronic population. The approximation works better for the quasiclassical regime with large vibrational amplitude and weak coupling.

Detuning of the electronic and vibrational frequencies slows down the relaxation of the electronic population oscillation. An increase of the initial vibrational energy qualitatively changes the evolution of the electronic population from continuous oscillation to beats that reflect relaxation. The rate of the relaxation drops with increasing vibrational amplitude. It is found that several dynamical variables oscillate with the same rate and relax at the same time. Quantum expectation values, such as the vibrational coordinate dispersion not included into the primary calculation scheme, preserve the dynamics during the silent period, when a single vibrational mode acts as a quasi heat bath and induces electronic population dephasing over a finite time. The relaxation of the electronic population and a noticeable growth of the vibrational coordinate dispersion originate from splitting of the vibrational wave function into two wavepackets that oscillate independently and correlate with different electronic states. The original Gaussian wavepacket transforms into a nonclassical state with large width. The vibrational energy flows between its quasiclassical and purely quantum components. Relaxation of the oscillation of the overall vibrational energy is compensated by growth in the oscillation of the vibrational coordinate dispersion.

The vibrational energy contains more quasiclassical energy at the initial and revival times and less quasiclassical energy during the silent time interval between the revivals. At the time of revivals the vibrational subsystem comes into a quasiclassical state. By analogy, the electronic energy flows between its quasiclassical and purely quantum components. The electronic energy contains less quasiclassical contribution at revival times and more quasiclassical contribution during the silent period. The interaction with the vibrational mode drives the electronic subsystem into a mixed state and reduces the length of the Bloch vector. In the middle of the silent interval, the electronic subsystem comes into a coherent state. This qualitative process is reflected in the time evolution of the squared length of the Bloch vector, the sum of the electronic dispersions, and the fidelity of the electronic subsystem, which are all linearly dependent.

Time dependence of the sums of the electronic and vibrational dispersions, and the 
electron-vibrational correlation oscillate with a low frequency corresponding to the rate of relaxation and dephasing of the fast first order dynamical variables. The extrema of the sums of the electronic and vibrational dispersions correspond to the fastest change in the electron-vibrational correlation and vice versa. Oscillations of the dispersions of the electronic and vibrational operators occur with the same frequencies. The correlation between the electronic and vibrational subsystems grows, oscillates, relaxes, and reappears similarly to the population inversion. Work in progress extends the reported results toward more accurate calculations and descriptions of molecular charge and excitation transfer observed in ultrafast optical experiments.

\section{Acknowledgments}

The research was supported by NSF, CAREER Award CHE-0094012. OVP is a Camille and Henry Dreyfus New Faculty and an Alfred P. Sloan Fellow. DSK thanks A. Piryatinski for fruitful discussions.

1 A. H. Zewail, J. Phys. Chem. A 1045660 (2000); Science 2421645 (1988).

2 V. Balzani and F. Scandola, Supramolecular Photochemistry (Ellis Horwood, Chichester, 1991).

3 H. van Ameroningen, L. Vakunas, and R. van Grondelle, Photosynthetic Excitons (World Scientific, 2000).

4 J. E. Monat, J. H. Rodriguez, J. K. McCusker, J. Phys. Chem. A 1067399 (2002).

5 W. Stier and O. V. Prezhdo, J. Phys. Chem. B 1068047 (2002); Isr. J. Chem. 42 213-224 (2003); J. Mol. Struct. (Theochem.) 63033 (2003); W. Stier, W. R. Duncan, and O. V. Prezhdo, Adv. Mat., in press.

6 C. Brooksby, O. V. Prezhdo, and P. J. Reid, J. Chem. Phys. 1184563 (2003); ibid 1199111 (2003).

7 J. Deisenhofer and H. Michel, Annu. Rev. Biophys. Biophys. Chem. 20, 247-266 (1991).

8 R. Uberna, M. Khalil, R. M. Williams, J. M. Papanikolas, and S. R. Leone, J. Chem. Phys. 1089259 (1998); T. W. Marin, B. J. Homoelle, K. G. Spears, J. T. Hupp, and L. O. Spreer, J. Phys. Chem. A 1061131 (2002). 
9 G. D. Scholes, X. J. Jordanides, G. R. Fleming, J. Phys. Chem. B 1051640 (2002).

10 M. R. Wasielewski, Chem. Rev. 92, 345 (1992); W. B. Davis, M. A. Ratner, M. R. Wasilewski, Chem. Phys. 281333 (2002). A. S. Lukas, P. J. Burchard, M. R.Wasilewski, J. Chem. Phys. A 1062074 (2002). M. R. Wasielewski, in: Photochemical Energy Conversion, Eds. J. Norris and D. Meisel (Elsevier, Amsterdam, 1989) p. 135; M. R. Wasielewski, Tetrahedron 45, 4785 (1989).

11 A. T. Yeh, C. V. Shank, J. K. McCusker, Science 289935 (2000); D. Kuciauskas, J. E. Monat, R. Villahermosa, H. B. Gray, N. S. Lewis, J. K. McCusker, J. Phys. Chem. B 1069347 (2002).

12 R. N. Zare, Science 2791875 (1998).

13 M. Lim and R. M. Hochstrasser, J. Chem. Phys. 1157629 (2001); P. Hamm, M. Lim, W. F. DeGrado, and R. M. Hochstrasser, J. Chem. Phys. 1121907 (2000); M. T. Zanni, M. C. Asplund, and R. M. Hochstrasser, J. Chem. Phys. 1144579 (2001).

14 B. I. Stepanov and V. P. Gribkovskii, Theory of luminescence; ed. S. Chomet (London, Iliffe, 1968).

15 D. J. Tannor, Introduction to Quantum Mechanics: A Time-Dependent Perspective, (University Science Press, Sausalito, 2001).

16 S. Mukamel, Principles of Nonlinear Optical Spectroscopy (Oxford University Press, New York, 1995).

17 S. Tretiak, A. Saxena, R. L. Martin, and A. R. Bishop, J. Chem. Phys. 115699 (2001).

18 S. Tanaka, S. Volkov, and S. Mukamel J. Chem. Phys. 1183965 (2003); S. Tanaka and S. Mukamel, ibid, 1161877 (2002)

19 K. A. Merchant, D. E. Thompson, and M. D. Fayer, Phys. Rev. A 65023817 (2002); D. E. Thompson, K. A. Merchant, and M. D. Fayer, J. Chem. Phys. 115317 (2001).

20 O. Golonzka, M. Khalil, N. Demirdöven, and A. Tokmakoff, J. Chem. Phys. 11510814 (2001); M. Khalil, N. Demirdven, and A. Tokmakoff, Phys. Rev. Lett. 90, 047401 (2003); N. Demirdöven, M. Khalil, and A. Tokmakoff, Phys. Rev. Lett. 89, 237401 (2002).

21 J. D. Hybl, A. A. Ferro, and D. M. Jonas, J. Chem. Phys. 1156606 (2001).

22 N. F. Sherer, R. Carlson, A. Matro, M. Du, A. L. Ruggiero, V. Romero-Rochin, J. A. Cina, G. R. Fleming, and S. A. Rice, J. Chem. Phys. 951487 (1991).

23 N. F. Sherer, A. Matro, R. J. Carlson, M. Du, L. D. Ziegler, J. A. Cina, and G. R. Fleming, J. Chem. Phys. 964180 (1992).

24 J. A. Cina, D. S. Kilin, and T. S. Humble, J. Chem. Phys. 118, 46 (2003). 
25 I. Sh. Averbukh, M. Shapiro, C. Leichtle and W. P. Schleich, Phys. Rev. A 592163 (1999).

26 R. A. Marcus, J. Chem. Phys. 24, 966 (1956); Rev. Mod. Phys. 65, 599 (1993); R. A. Marcus and N. Sutin, Biochim. Biophys. Acta 811, 265 (1985).

27 Th. Förster, Delocalized excitation and excitation transfer in: Modern Quantum Chemistry, Ed. O. Sinanoglu, (Academic, NY, 1965).

28 M.V. Volkenshtein, M.A. Eliashevich, B.I. Stepanov, Oscillations of Molecules, (Moskow, 1947).

29 V. May and O. Kühn, Charge and Energy Transfer Dynamics in Molecular Systems, (WileyVCH, 2000).

30 O. V. Prezhdo and P. J. Rossky, J. Chem. Phys. 1075963 (1997); B. J. Schwartz, E .R. Bittner, O. V. Prezhdo, and P. J. Rosski, J. Chem. Phys. 1095942 (1996).

31 G. C. Schatz and M. A. Ratner, Quantum mechanics in chemistry, (Englewood Cliffs, NJ, Prentice Hall, 1993).

32 E. J. Heller, J. Chem. Phys. 621544 (1975).

33 Y. J. Yan and S. Mukamel, J. Chem. Phys. 885735 (1988); ibid 895160 (1988); J. Grad, Y. J. Yang, A. Haque, and S. Mukamel, ibid 863441 (1987); J. Grad, Y. J. Yan, and S. Mukamel, Chem. Phys. Lett. 143291 (1987); S. Mukamel, J. Phys. Chem. 883185 (1984).

34 W. H. Miller, J. Chem. Phys. 621899 (1975).

35 R. P. Feynmann, Quantum Mechanics and Path Integrals (NY, McGrow-Hill, 1965).

36 J. C. Tully, in Classical and Quantum Dynamics in Condenced Phase Simulations, ed. B. J. Berne, G. Cicotty, and D. F. Coker (World Scientific, 1998).

37 D. F. Coker, in Computer Simulations in Classical Physics, ed. M. P. Allen and D. J. Tildesley (Kluwer, 1993).

38 C. J. Smallwood, W. B. Bosma, R. E. Larsen, and B. J. Schwartz, J. Chem. Phys. 11911263 (2003); R. E. Larsen and B. J. Schwartz, ibid 1197672 (2003).

39 S. Karabunarliev and E. R. Bittner, J. Chem. Phys. 1193988 (2003).

40 O. V. Prezhdo and Yu. V. Pereversev, J. Chem. Phys. 1136557 (2000).

41 O. V. Prezhdo, J. Chem. Phys. 1172995 (2002).

42 O. V. Prezhdo and Yu. V. Pereverzev, J. Chem. Phys. 1164450 (2002).

43 E. Pahl and O. V. Prezhdo, J. Chem. Phys. 1168704 (2002).

44 C. Brooksby, O. V. Prezhdo, Chem. Phys. Lett. 378533 (2003); J. Mol. Struct. (Theochem) 63045 (2003). 
45 G. C. Wick, Phys. Rev. 80268 (1950); R. P. Feynman, Phys. Rev. 76749 (1949); F. J. Dyson, Phys. Rev. 75286 (1949).

46 A. Leggett, Rev. Mod. Phys. 591 (1987).

47 P. Meystre and M. Sargent III, Elements of Quantum Optics (Springer, 1990). K. Blum, Density Matrix, Theory and Applications (Plenum, 1981); Y. Yamamoto, Mesoscopis Quantum Optics (Wiley, 1999); R. Puri, Mathematical Methods in Quantum Optics (Springer, 2000); S. M. Barnett and P. M. Radmore, Methods in Theoretical Quantum Optics (Clarendon, 1997);

48 L. Allen and J. H. Eberly, Optical Resonance and Two-Level Atoms (Wiley-VCH, 1975).

49 E. T. Jaynes and F. W. Cummings, Proc. IEEE 5189 (1963)

50 B. W. Shore and P. L. Knight, J. Mod. Optics 401195 (1993).

51 D. Bonatosos, Phys. Rev. A 47, 3448 (1993); S. Yu et.al., Phys. Rev. A 52, 2585 (1995); N. Alvarez M. and V. Hussin, J. Math. Phys. 43, 2063 (2002).

52 J. R. Ackerhalt and K. Rzazewski, Phys. Rev. A 122549 (1975).

53 M. O. Scully and M. S. Zubairu, Quantum Optics, (Cambridge, 1997).

54 F. W. Cummings, Phys. Rev. 140 A1051 (1965).

55 J. H. Eberly, N. B. Narozhny, and J. J. Sanchez-Mondragon, Phys. Rev. Lett. 441323 (1980).

56 G. P. Berman, E. N. Bulgakov, and D. D. Holm, Crossover-Time in Quantum Boson and Spin Systems (Springer, 1994).

57 H. J. Carmichael, Statistical Methods in Quantum Optics 1, Master Equations and Fokker-Plank Equations (Springer, 1999).

58 A. G. Redfield, Phys. Rev. 98, 1787 (1955); IBM J. Res. Dev. 1, 19 (1957); Adv. Magn. Reson. 1,1 (1965).

59 D. Kilin, M. Schreiber, J. Lumin. 92, 13 (2001).

60 W. T. Pollard and R. A. Friesner, J. Chem. Phys. 100, 5054 (1994).

61 M. I. Sakola, A. R. Bishop, V. M. Kenkre, and S. Raghavan, Phys. Rev. B 52 R3824 ('95).

62 P. A. Apanasevich, S. Ya. Kilin, and A. P. Nisovtsev, J. Appl. Spectr., 471213 (1987); S. Ya. Kilin and T. B. Krinitskaya, Phys. Rev. A 483879 (1993); D. Mogilevtsev and S. Kilin, Phys. Rev. A 67, 023815 (2003).

63 T. Holstein, Ann. Phys. 8325 (1959).

64 R. Agarwal, B. S. Prall, A. H. Rizvi, M. Yang, and G. R. Fleming, J. Chem. Phys. 1166243 (2002). 
TABLE I: Electronic and vibrational energies partitioned into quantum and classical contributions. The energies are given in the ladder, phase space and dispersion operator representations. In both electronic and vibrational subsystems the energy flows between the quantum and quasi-classical contributions.

\begin{tabular}{lll}
\multicolumn{3}{c}{ Energy } \\
\hline total & quasiclassical & pure quantum \\
\hline
\end{tabular}

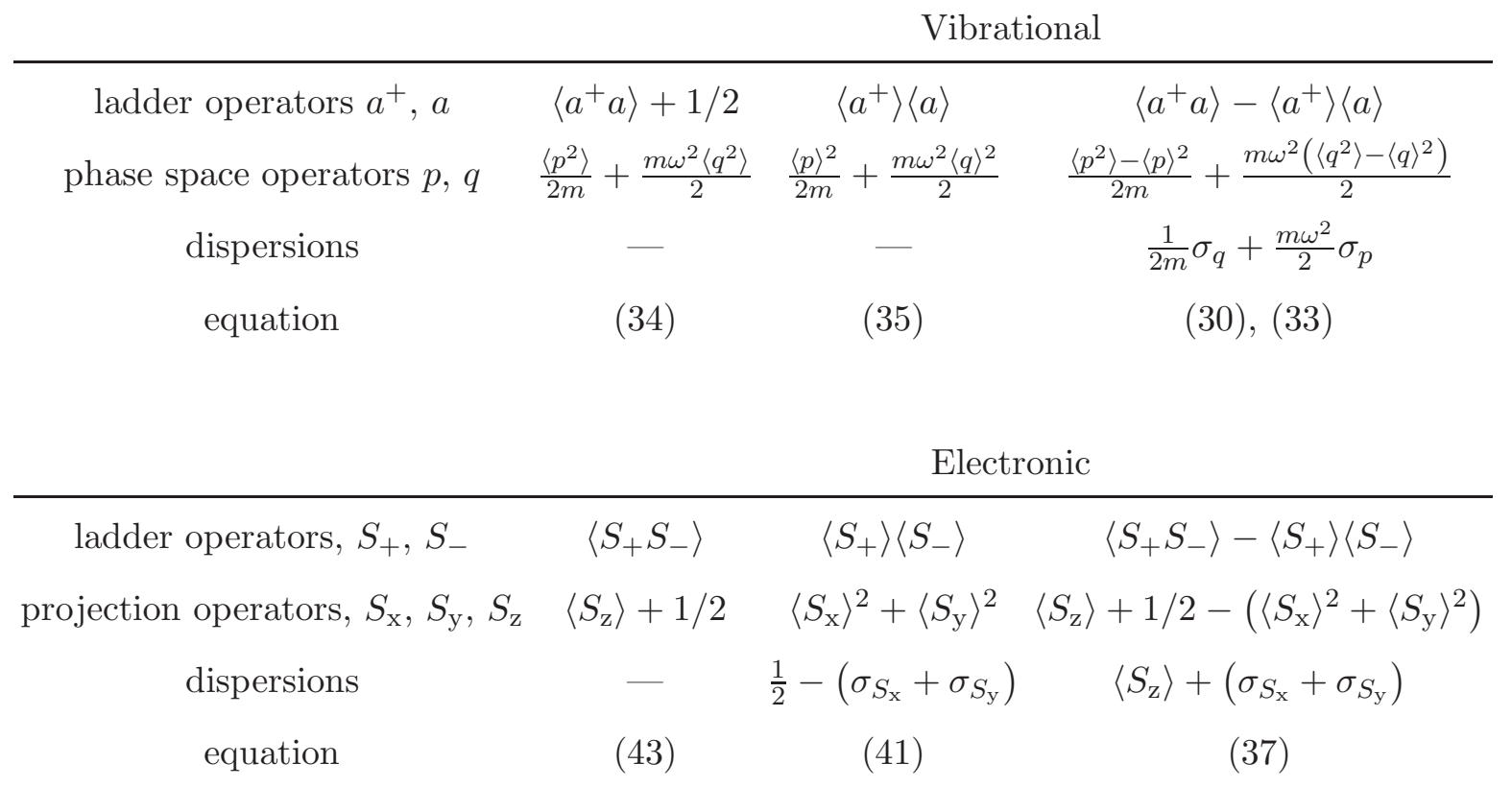


TABLE II: Key characteristics of the electronic subsystem: Bloch vector squared $\left(R^{2}\right)$, pure quantum energy $\left(E_{\mathrm{el}}^{\text {quant }}\right)$, and fidelity $\left(\operatorname{Tr} \rho^{2}\right)$ are represented by the expectation values of the ladder, projection and dispersion operators. These characteristics obey the equalities for pure states and the inequalities for mixed states.

$$
R^{2} \leq 1 / 4 \quad E_{\text {el }}^{\text {quant }} \geq 0 \quad \operatorname{Tr} \rho^{2} \leq 1
$$

projections, $S_{\mathrm{x}}, S_{\mathrm{y}}, S_{\mathrm{z}}$

dispersions

$$
1 / 2+\left\langle S_{z}\right\rangle^{2}-\sigma_{S_{\mathrm{x}}}-\sigma_{S_{\mathrm{y}}}
$$

$$
\left\langle S_{+} S_{-}\right\rangle-\left\langle S_{+}\right\rangle\left\langle S_{-}\right\rangle
$$$$
1+2\left\langle S_{+}\right\rangle\left\langle S_{-}\right\rangle-2\left\langle S_{+} S_{-}\right\rangle\left\langle S_{-} S_{+}\right\rangle
$$$$
\left\langle S_{z}\right\rangle+1 / 2-\left\langle S_{\mathrm{x}}\right\rangle^{2}-\left\langle S_{\mathrm{y}}\right\rangle^{2} 1 / 2-2\left\langle S_{\mathrm{z}}\right\rangle^{2}+2\left(\left\langle S_{\mathrm{x}}\right\rangle^{2}+\left\langle S_{\mathrm{y}}\right\rangle^{2}\right)
$$

$\left\langle S_{\mathrm{z}}\right\rangle+\sigma_{S_{\mathrm{x}}}+\sigma_{S_{\mathrm{y}}}$

$$
1 / 2-2\left\langle S_{\mathrm{z}}\right\rangle^{2}+1-2\left(\sigma_{S_{\mathrm{x}}}+\sigma_{S_{\mathrm{y}}}\right)
$$




\section{Figure Captions}

FIG. 1 Electronic states and state-specific potential energy curves as functions of the vibrational coordinate of the dimer described by the Hamiltonian (12), appearing in the theory of molecular excitons and also known as the Marcus model. The electronic states are separated by the energy gap $\varepsilon=\hbar \Omega$. The equilibrium positions in the vibrational coordinates of the two electronic states are displaced by the value specified in Eq. (11) and related to the nuclear reorganization $\left(\bar{\alpha}+\bar{\alpha}^{*}\right) / 2$.

FIG. 2 The expectation value of the population inversion operator $S_{z}$ as a function of time computed exactly, solid line, and approximately by Eq. (20), dotted line. (a) illustrates the quasi-classical case of a weak electron-phonon coupling $g=0.025$ and a large amplitude vibrational motion $\left\langle a^{+} a\right\rangle(t=0)=49$. (b) corresponds to a deep quantum case of a strong coupling $g=0.25$ and a smaller amplitude vibration $\left\langle a^{+} a\right\rangle(t=0)=9 .\left\langle S_{z}\right\rangle_{t=0}=-1 / 2$ and $\delta=0$ in both cases. Good agreement between the exact and approximate solutions is observed during the first several periods of the population inversion. For both types of initial conditions, the approximate solution correctly reproduces the dephasing envelope describing the decay in the amplitude of the oscillation of the electronic populations.

FIG. 3 Population inversion $t_{+}^{-1}$ and relaxation $t_{-}^{-1}$ rates, Eq. (23), labeled by " +" and "-", as functions of (a) detuning $\delta$, and (b) amplitude $\bar{\alpha}$, Eq. (11). The remaining parameters are kept constant at quasi-classical $\left\langle a^{+} a\right\rangle=49, g=0.025$ (solid line) and quantum $\left\langle a^{+} a\right\rangle=9, g=0.25$ (dashes) values used in Fig. 2. The dependence of the inversion relaxation rate on the amplitude of vibrational motion displays branching in (b). The inversion $t_{+}^{-1}$ and relaxation $t_{-}^{-1}$ rates coincide for small values of the vibrational amplitudes $\bar{\alpha}$. The branches split at $\bar{\alpha}=1$.

FIG. 4 Evolution of the sum of the population inversion $2\left\langle S_{z}\right\rangle$ and the electron-phonon correlation $\bar{\alpha}^{-1}\langle\beta\rangle$. The purely real $2\left\langle S_{\mathrm{z}}\right\rangle$ and imaginary $\bar{\alpha}^{-1}\langle\beta\rangle$ functions shown in (a) are dynamic conjugates in the sense of Eq. (16) with $n=0$. The absolute value $\left|\left\langle 2 S_{\mathrm{z}}+\bar{\alpha}^{-1} \beta\right\rangle\right|$ is shown in (b). The initial conditions and parameters are $\left\langle a^{+} a\right\rangle_{t=0}=9,\left\langle S_{z}\right\rangle_{t=0}=-1 / 2$, $g=0.25, \delta=0$, corresponding to Fig. 2(b). The population inversion $S_{\mathrm{z}}$ and the electron-phonon correlation $\beta$ oscillate and dephase with the same rate. The amplitude $\left|\left\langle 2 S_{\mathrm{z}}+\bar{\alpha}^{-1} \beta\right\rangle\right|$ displays smooth dephasing dynamics without the quick oscillations. 
FIG. 5 Key characteristics of the vibrational subsystem. (a): Dispersions $\sigma_{q}, \sigma_{p}$ of the vibrational coordinate $q$ and momentum $p$ are shown by the solid and dotted lines, Eqs. (31) and (32), respectively. The sum $\sigma_{q}+\sigma_{p}$ Eq. (33), shown by the dashed line starts from the minimum uncertainty width and smoothly increases to its maximum value that is equal to the total energy of the system. The sum of dispersions serves as an envelope for the individual dispersions. The slow increase of the dispersion is due to the splitting of the original Gaussian wavepacket into two Gaussians that oscillate independently and pass through each other leading to the rapid oscillations of $\sigma_{q}$ and $\sigma_{p}$. (b): Shown are the total energy of the vibrational mode Eq. (34), solid line, together with its quasi-classical contribution, Eq. (35), dots, and the purely quantum contribution, Eq. (33), dashes. The vibrational energy flows between its quasiclassical and purely quantum contributions. At $t=0$ the quantum contribution equals zero-point energy, and the rest of the vibrational energy is quasiclassical. When the total vibrational energy stops oscillating, it is purely quantum. When the oscillation resumes, the vibrational energy is dominated by the classical contribution. The sum of dispersions in (a) is an alternative representation of the quantum energy in (b), dashed lines. The initial conditions and model parameters are the same as in Fig. 2(b). Note, that the phase of the vibrational energy oscillation in this figure is opposite to the phase of the electronic population in Fig. 2(b), due to conservation of the total electron-vibrational energy.

FIG. 6 (a) Time slices of the vibrational wavepacket in coordinate representation, (b) the expectation value of the vibrational coordinate $q,(\mathbf{c})$ and the expectation value of the population inversion $\left\langle S_{z}\right\rangle+1 / 2$. The initial conditions and model parameters are taken from Ref. [59]. Transfer of population between the two states affects the wavepacket dynamics. The original Gaussian wavepacket splits into two wavepackets correlated with each electronic state. As a result of the splitting the oscillation of the average vibrational coordinate decays. Most interestingly, the oscillation of the electronic population decays as well. The decay in the oscillation of the electronic population, also known as dephasing, typically occurs by coupling to a heat bath. Remarkably, a single vibrational mode acts as a quasi-heat bath and induces population dephasing over a finite time.

FIG. 7 Key characteristics of the electronic subsystem, cf. Fig. 6 for the vibrational subsystem. (a): Dispersions $\sigma_{S_{\mathrm{x}}}, \sigma_{S_{\mathrm{y}}}$ of the conjugate electronic operators $S_{\mathrm{x}}$ and $S_{\mathrm{y}}$ are 
shown by the solid and dashed lines, Eqs. (39) and (40), respectively. The sum $\sigma_{S_{\mathrm{x}}}+\sigma_{S_{\mathrm{y}}}-$ 1/4, Eq. (41) shown by the thick dot-dashed line starts from the maximum value of $1 / 4$, corresponding to a pure state, and varies more smoothly than the components $\sigma_{S_{\mathrm{x}}}, \sigma_{S_{\mathrm{y}}}$, creating an envelope. In contrast to the vibrational dispersions, Fig. 5, which increase due to the electron-vibrational interaction, the electronic dispersions decrease. (b): Shown are the total energy of the electronic subsystem $\left\langle S_{+} S_{-}\right\rangle$, Eq. (43), solid line, its quasiclassical contribution, Eq. (42), dots, and the purely quantum contribution, Eq. (44), dashes. The electronic and vibrational energies, this figure and Fig. 5, oscillate with opposite phases so that the total electron-vibrational energy remains constant. The electronic energy flows between its quasiclassical and purely quantum contributions. At $t=0$ both quantum and classical contributions equal zero since system is prepared in the state $\left\langle S_{\mathrm{z}}\right\rangle(0)=-1 / 2$ During the silent period, when the population inversion stops oscillating, the electronic energy contains the largest portion of the classical contribution, while the vibrational energy is purely quantum. The sum of dispersions in (a) is an alternative representation of the classical component of the electronic energy in (b), up to the linear transformation of Eq. (41). The initial conditions and model parameters are the same as in Fig. 2(b).

FIG. 8 Slowly oscillating combinations of the expectation values. The initial conditions and model parameters are the same as in Fig. 2(b). (a). Time dependence of the sums of the electronic and vibrational dispersions, solid and dashed lines, respectively. Surprisingly, the sum of the electronic dispersions, Eq. (41), gives the quasiclassical contribution to the electronic energy, Eq. (42), while the sum of vibrational dispersions gives the pure quantum contribution to the vibrational energy, Eq. (33). (b): Imaginary part of the correlation between the electronic and vibrational subsystems. The expectation value of the correlator Eq. (46) is purely imaginary, its magnitude is displayed on the $y$-axis. The electron-vibrational correlator starts at 0 and evolves on the slow time-scale of formation and dephasing of the electron-vibrational entanglement. The extrema of the sums of the electronic and vibrational dispersions in (a) correspond to the fastest change in the correlation in (b) and vice versa. The characteristics displaced in the figure are expressed in several ways in Table I.

FIG. 9 Trajectories of the Bloch vector, Bloch vector squared, and a relationship between the key characteristics of the electronic subsystem. (a): Bloch vector, Eq. (48), as a 
function of time during the first one and a half population inversions, for the same initial conditions and model parameters as in Fig. 2(b). The Bloch vector is defined by the three spin projections of the electronic two-level system $\left\langle S_{\mathrm{x}}\right\rangle,\left\langle S_{\mathrm{y}}\right\rangle,\left\langle S_{\mathrm{z}}\right\rangle$ and is shown together with a Bloch sphere of radius $1 / 2$. At $t=0$ the Bloch vector connects the origin and the south pole of the Bloch sphere. The Bloch vector revolves from the south pole to the north pole and changes in length. (b): The squared length of the Bloch vector as a function of time. $R^{2}=1 / 4$ at $t=0$, decreases at $0<\omega t<2$, and rises to a local maximum at $\omega t \simeq 6$. (c): Key characteristics of the electronic subsystem as functions of the horizontal projection of the Bloch vector, Eq. (50), for $\left\langle S_{z}\right\rangle=0,\left\langle S_{+} S_{-}\right\rangle=\left\langle S_{-} S_{+}\right\rangle=1 / 2$. Shown are the Bloch vector length squared Eq. (51), solid line, the sum of dispersions for the electronic subsystem Eq. (41), dot-dashes, the quantum contribution to the electronic energy Eq. (44), dots, and fidelity Eq. (47), dashes. The right edge, $\left\langle S_{\mathrm{x}}\right\rangle^{2}+\left\langle S_{\mathrm{y}}\right\rangle^{2}=0.25$, corresponds to pure states characterized by maximal fidelity, $\operatorname{Tr} \rho_{\mathrm{el}}^{2}=1$ and $R^{2}=1 / 4$. The left edge, $\left\langle S_{\mathrm{x}}\right\rangle^{2}+\left\langle S_{\mathrm{y}}\right\rangle^{2}=0$, describes the mixed state with minimal fidelity, $\operatorname{Tr} \rho_{\mathrm{el}}^{2}=1 / 2$ and $R^{2}=0$. Interaction with the bosonic mode drives the electronic mode from a pure to a mixed state. 


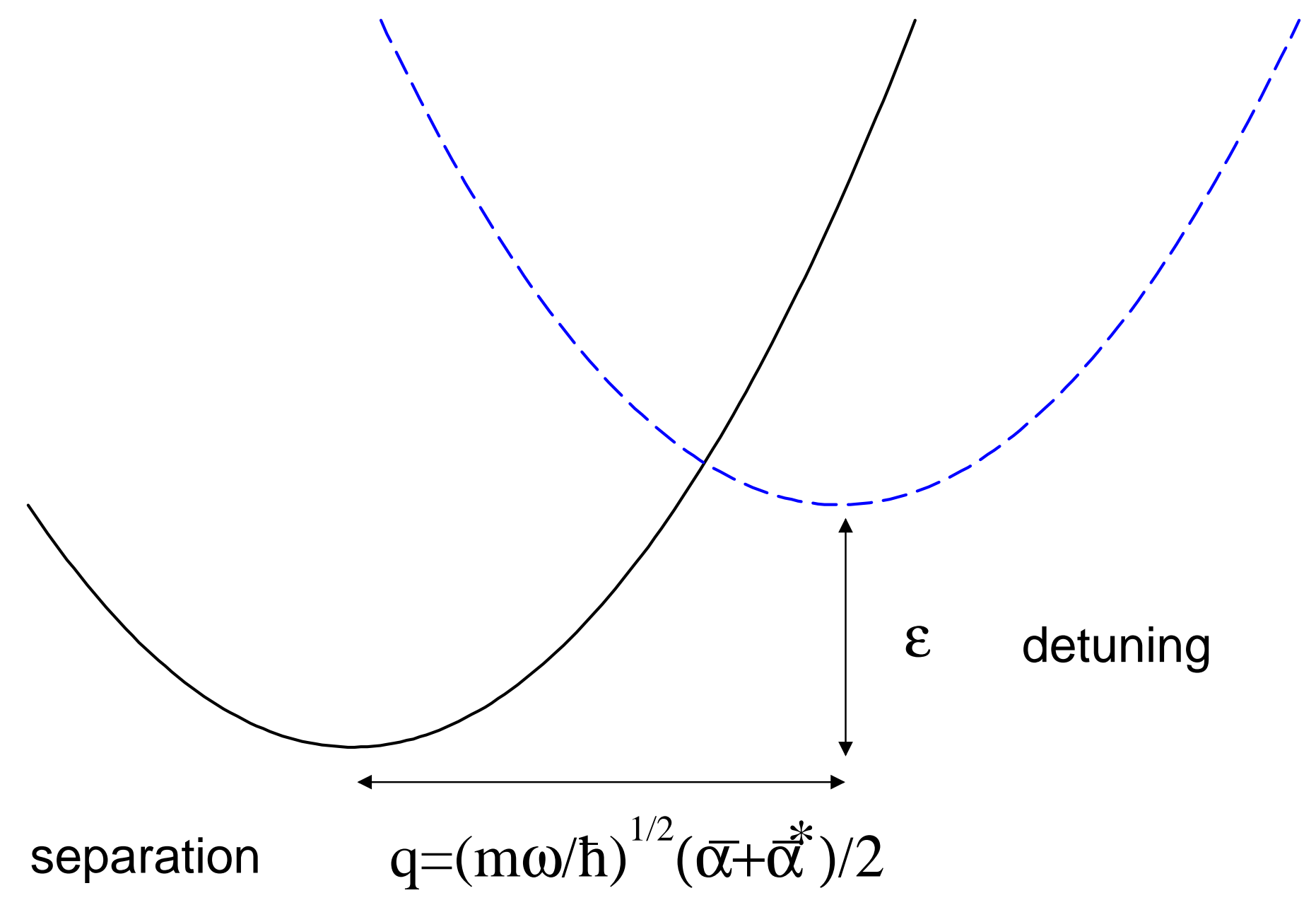



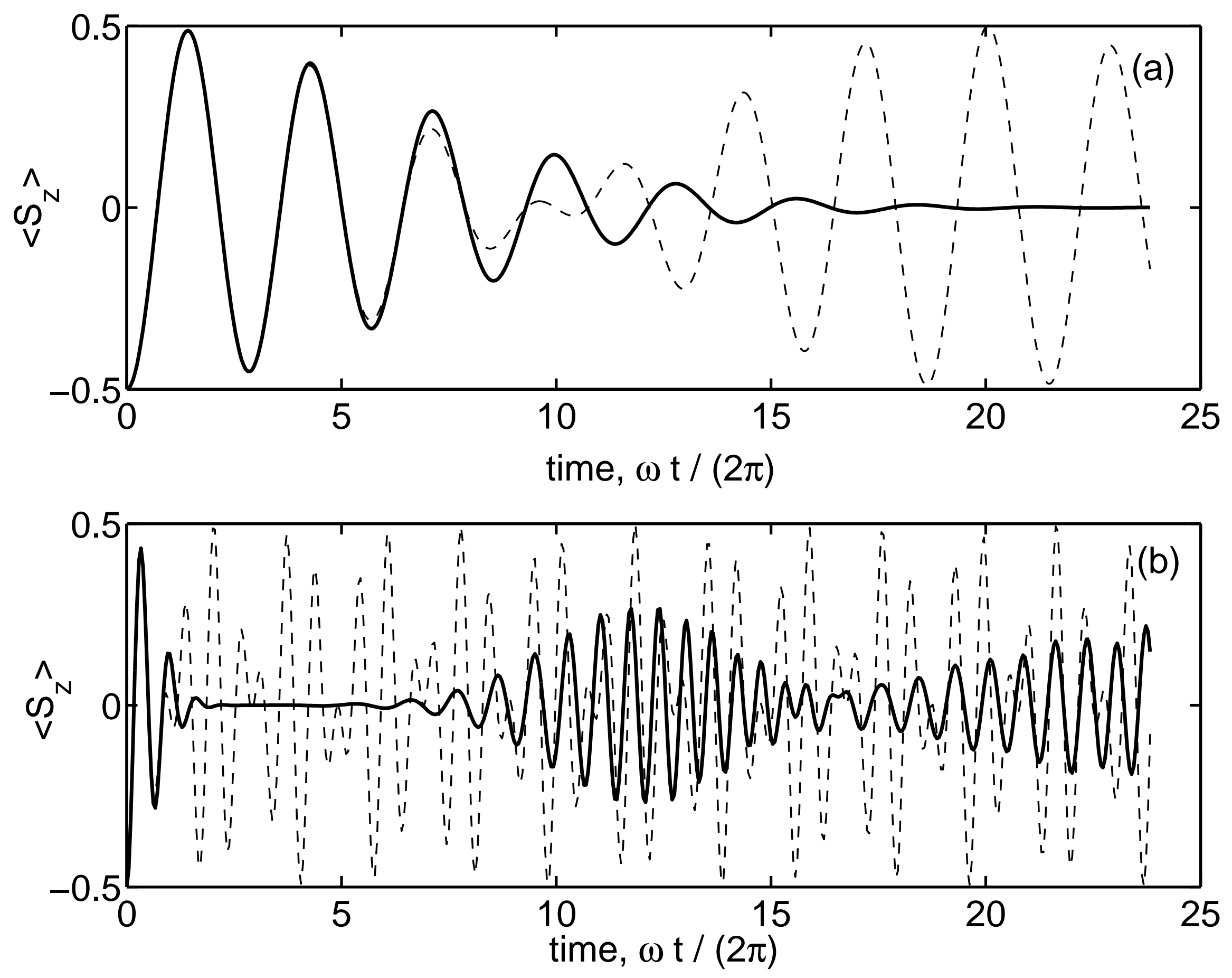

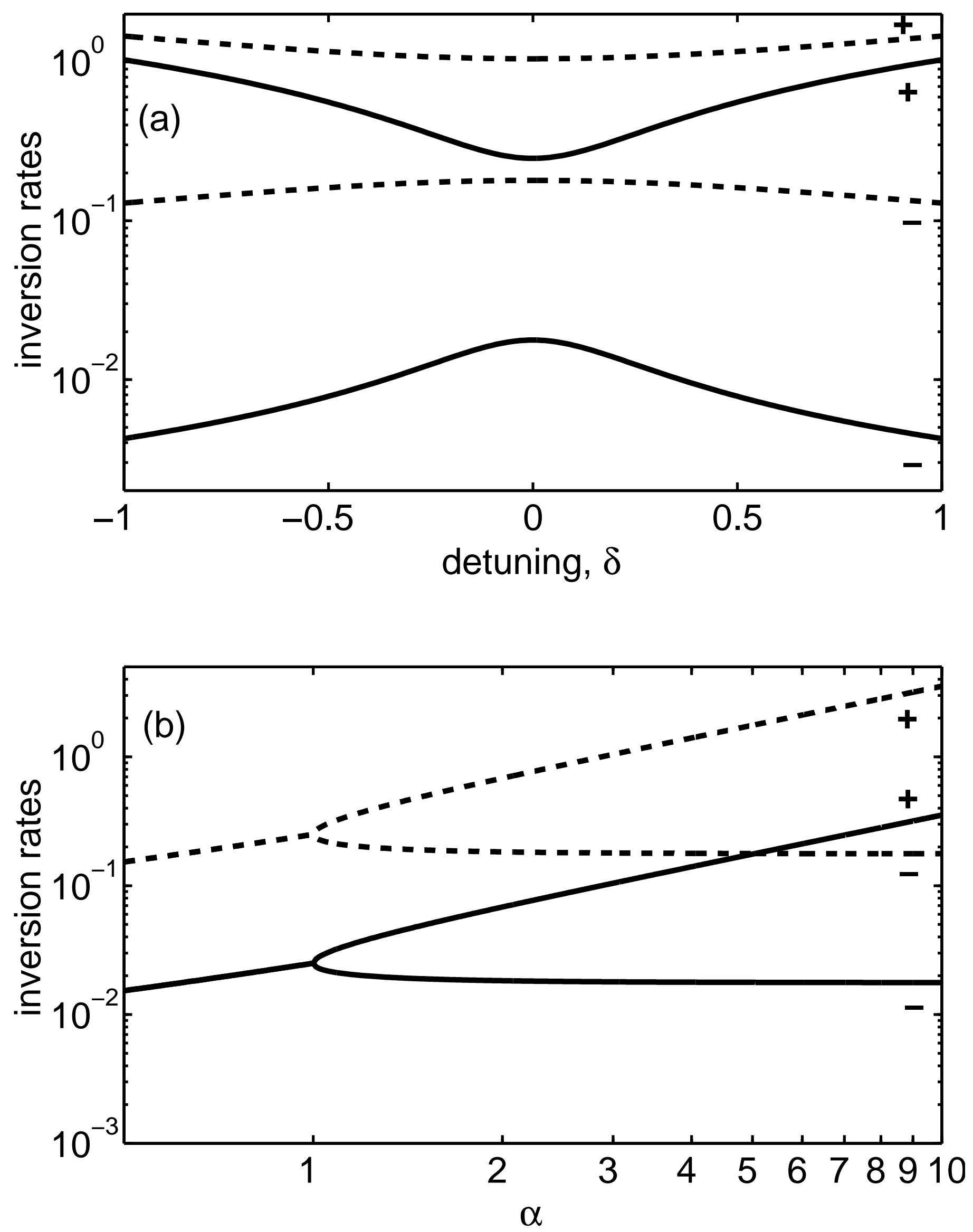

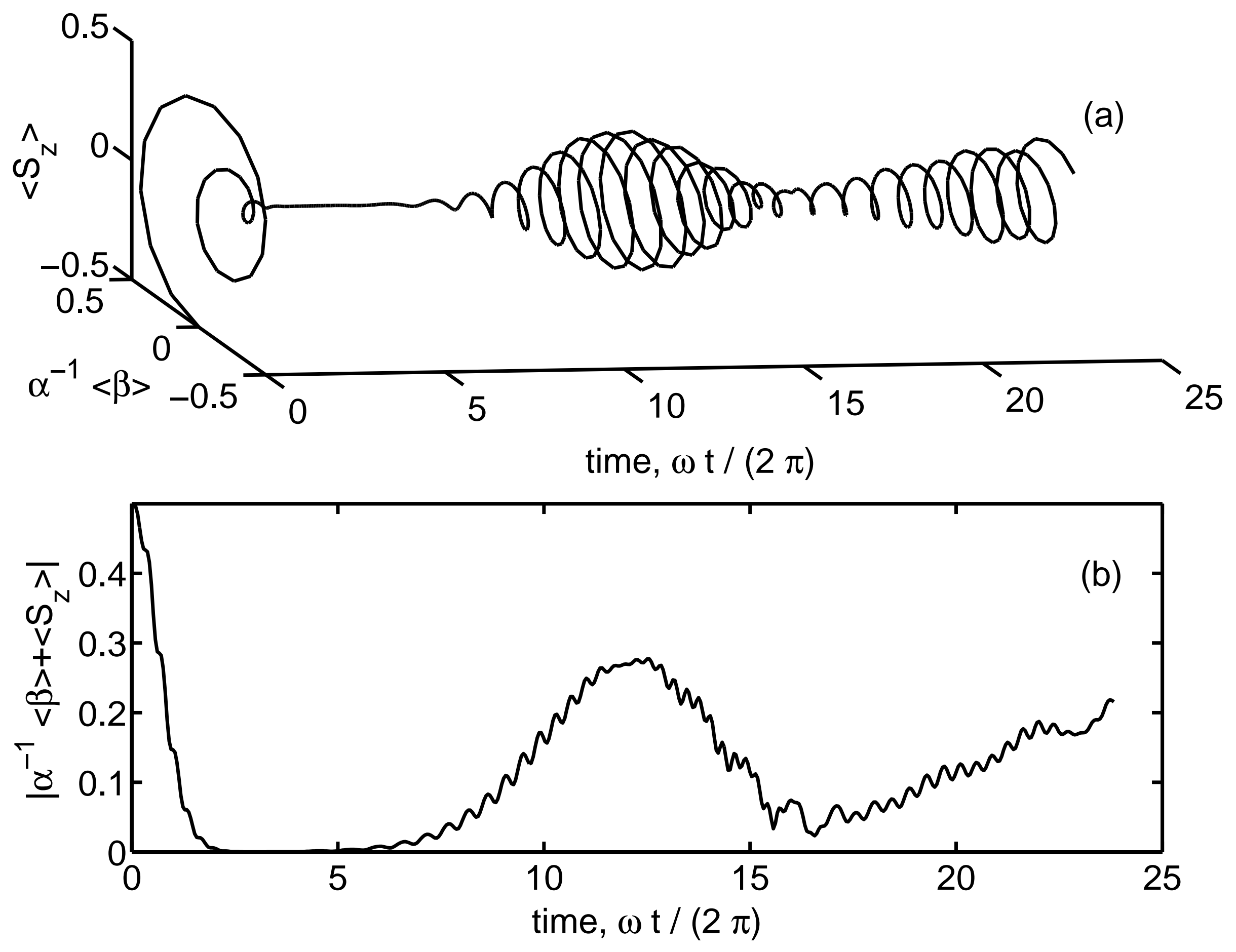

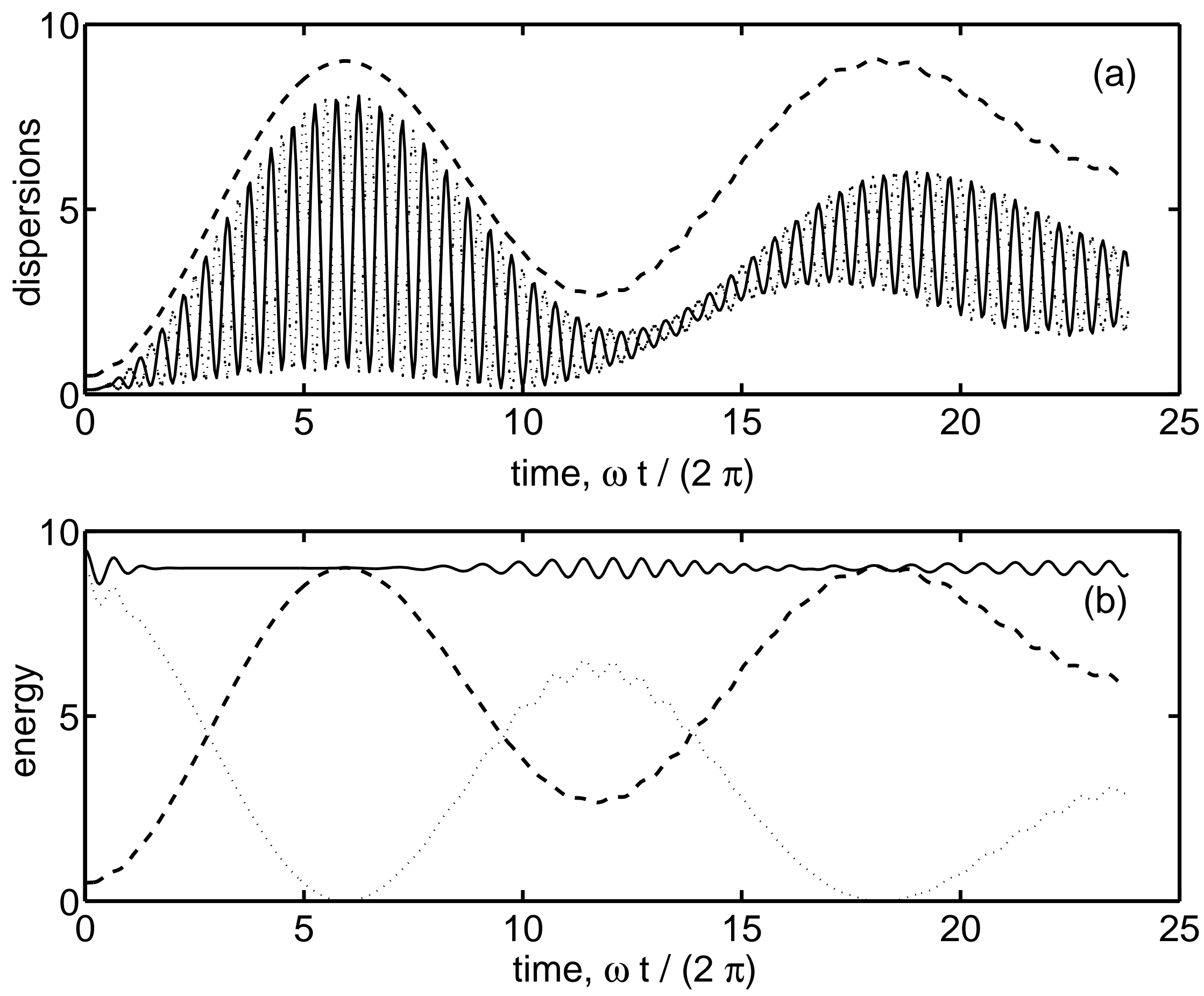


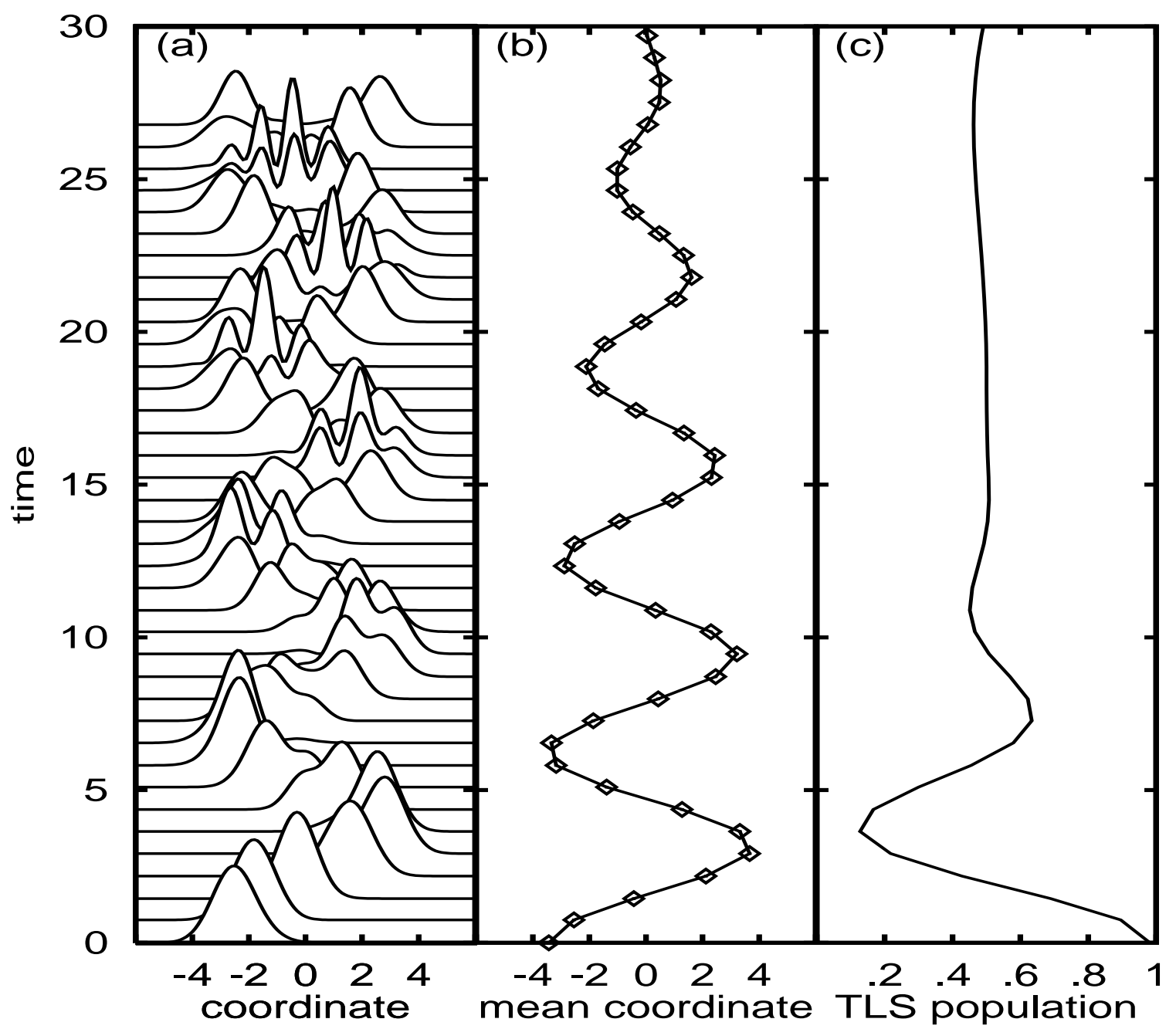



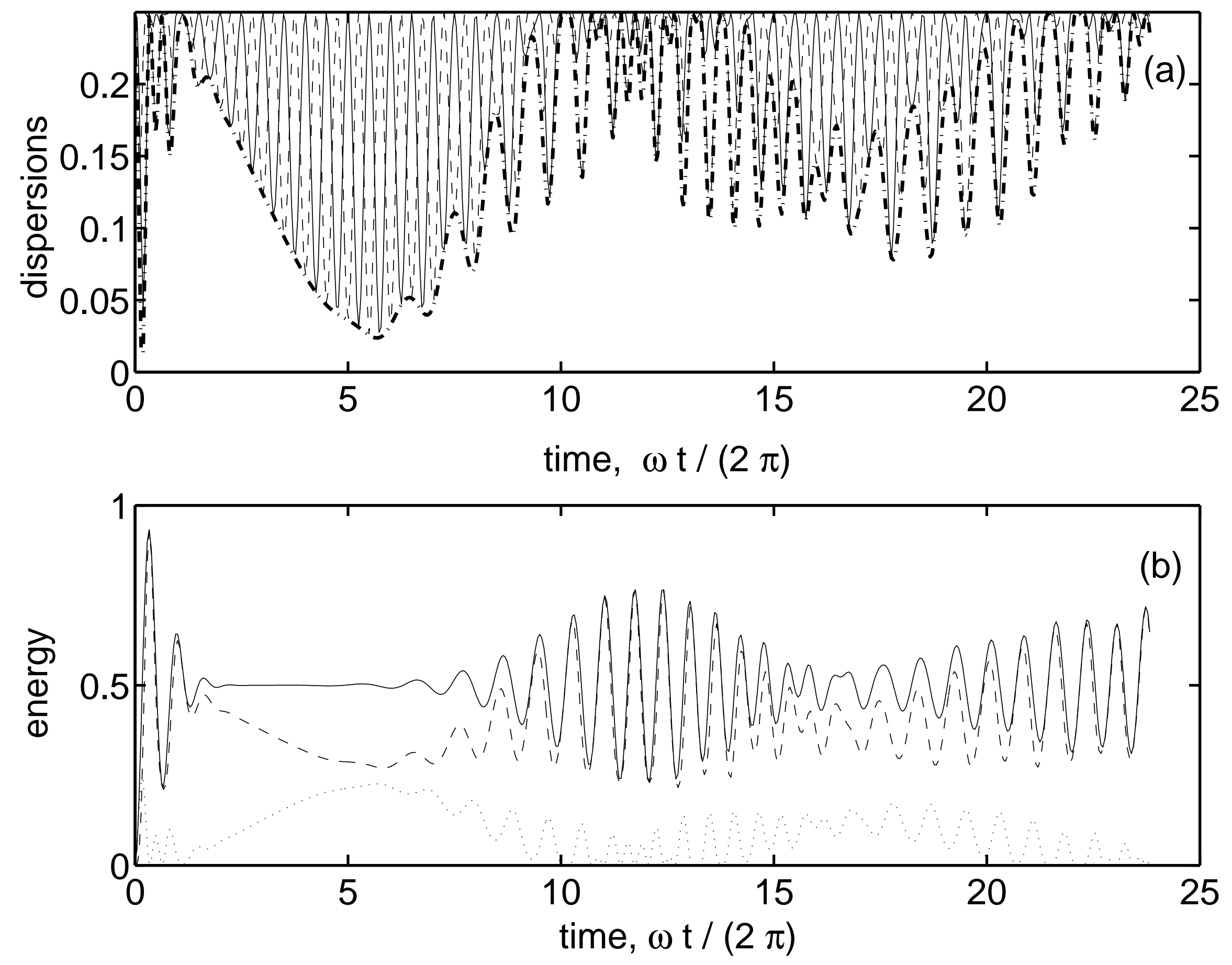


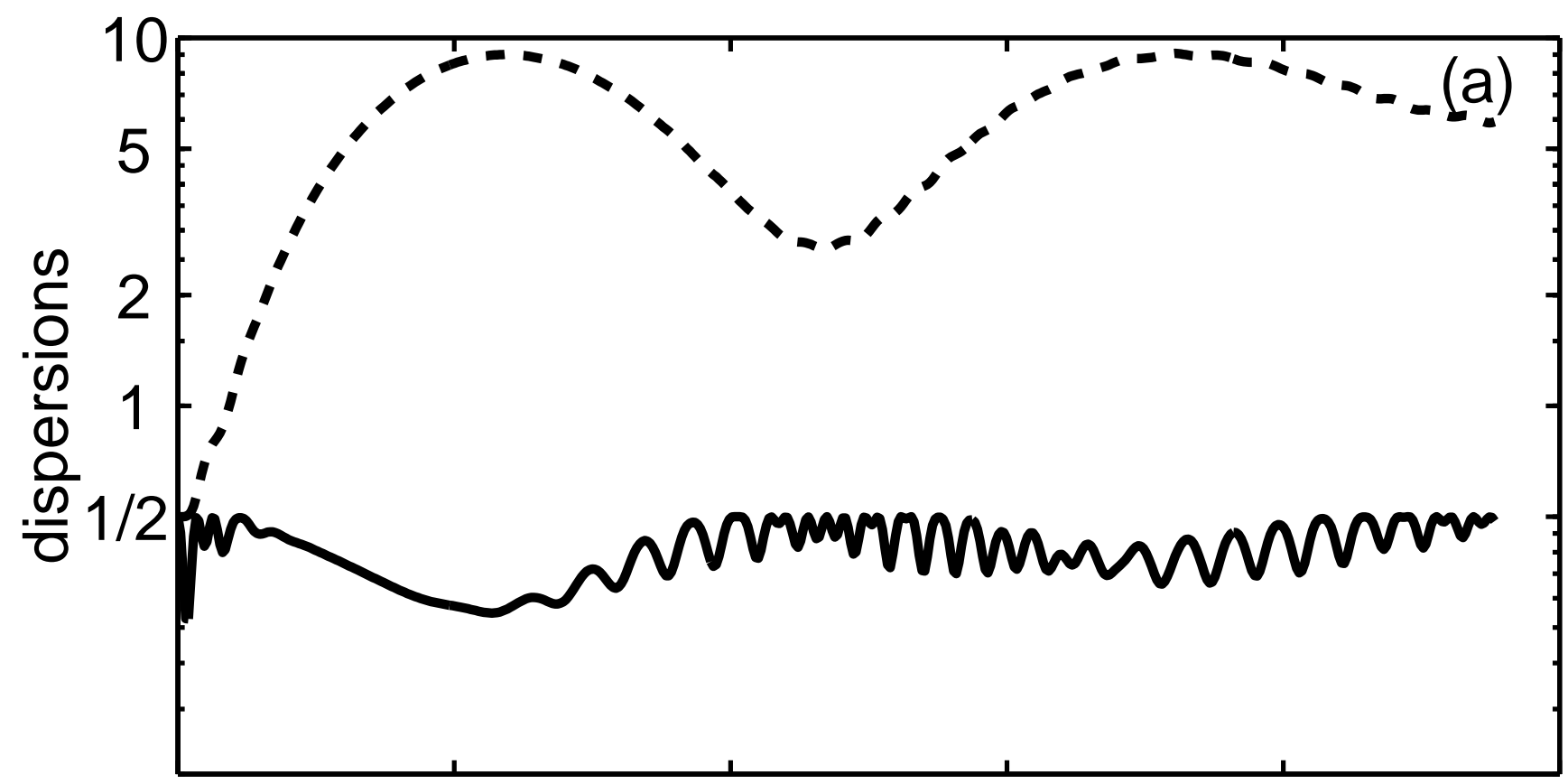




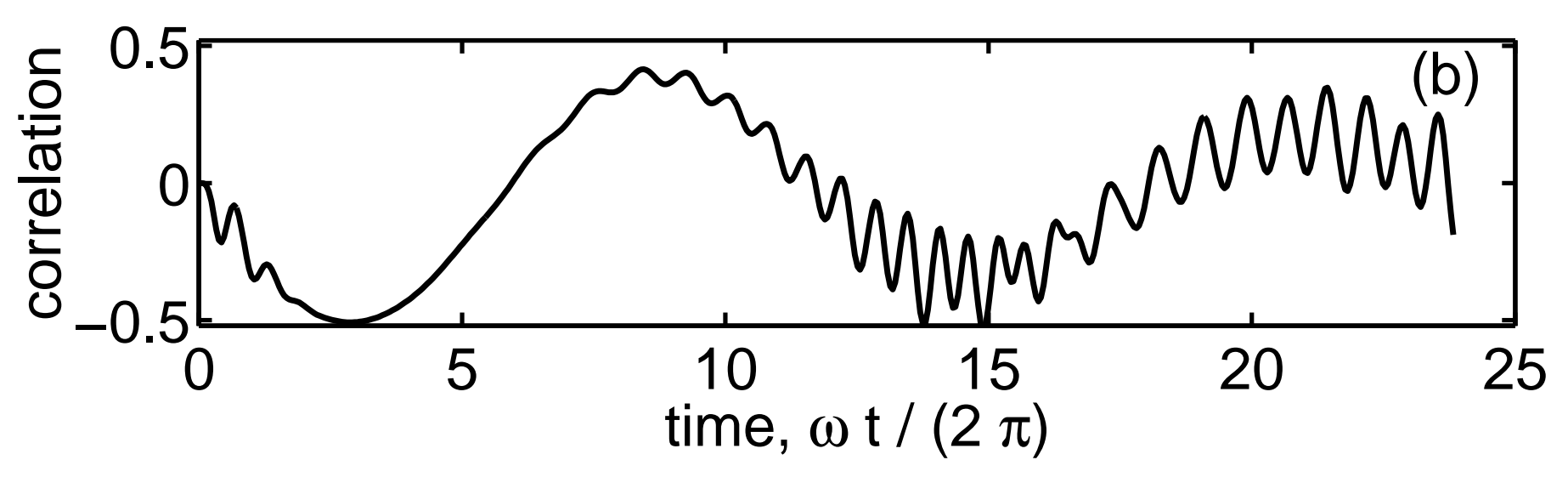




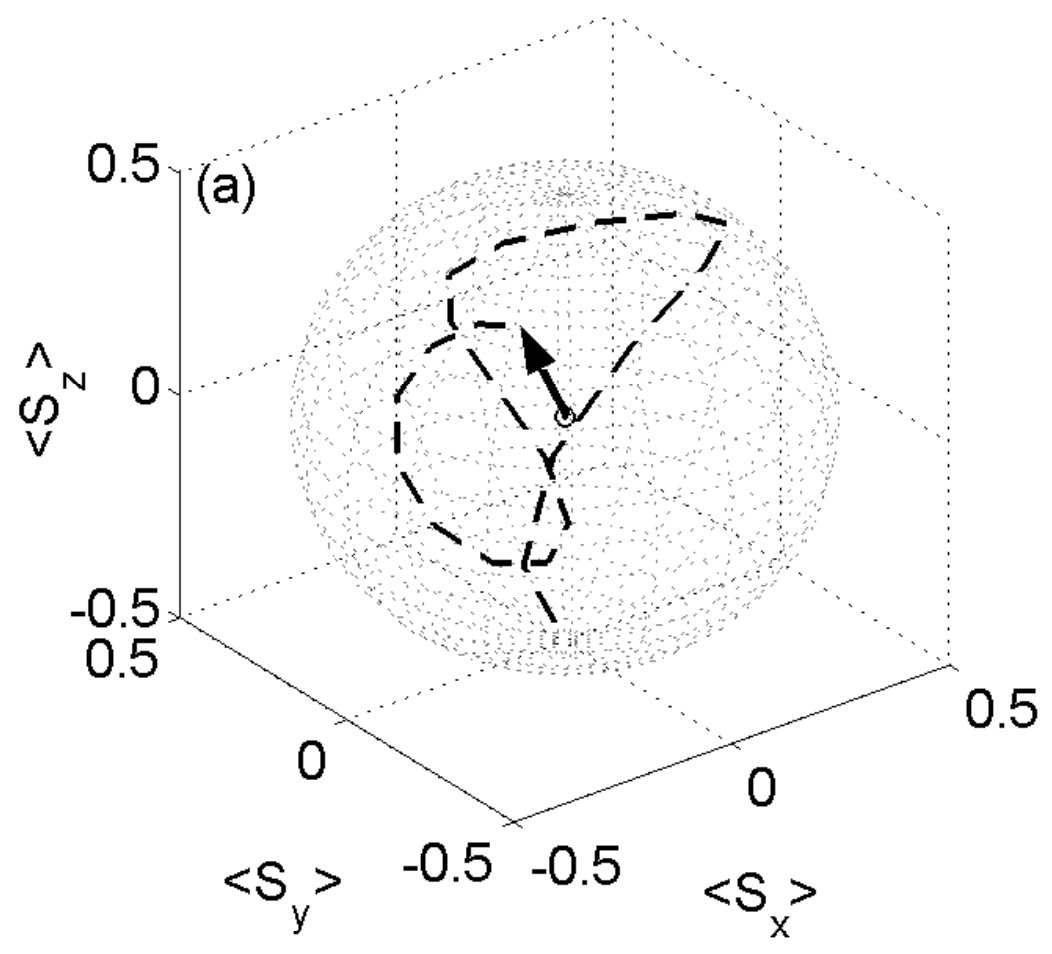




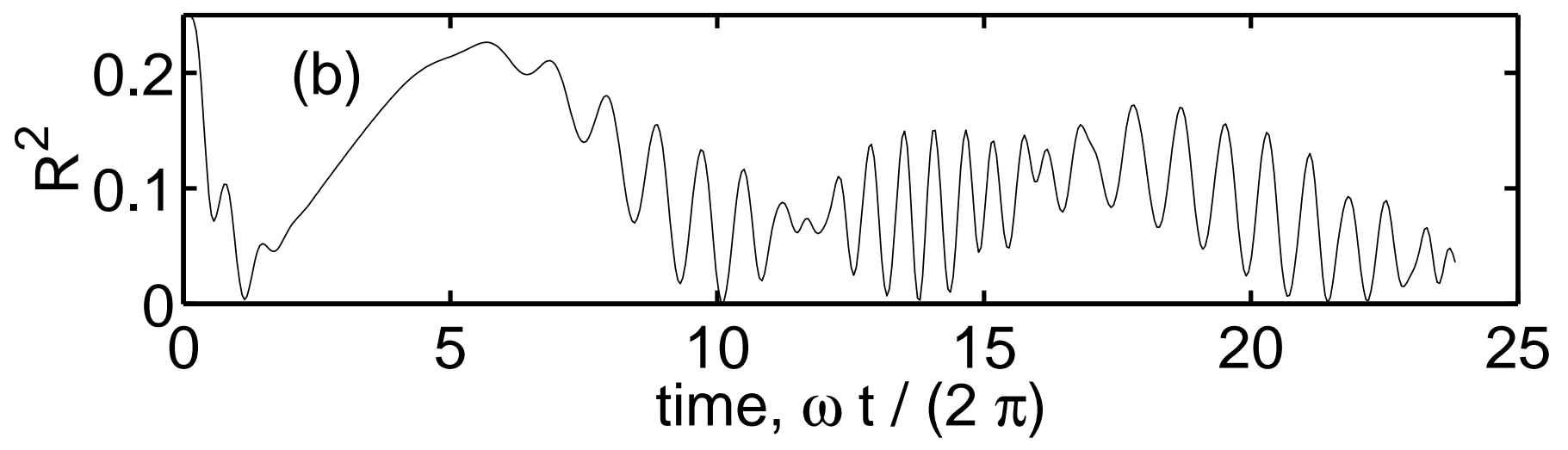




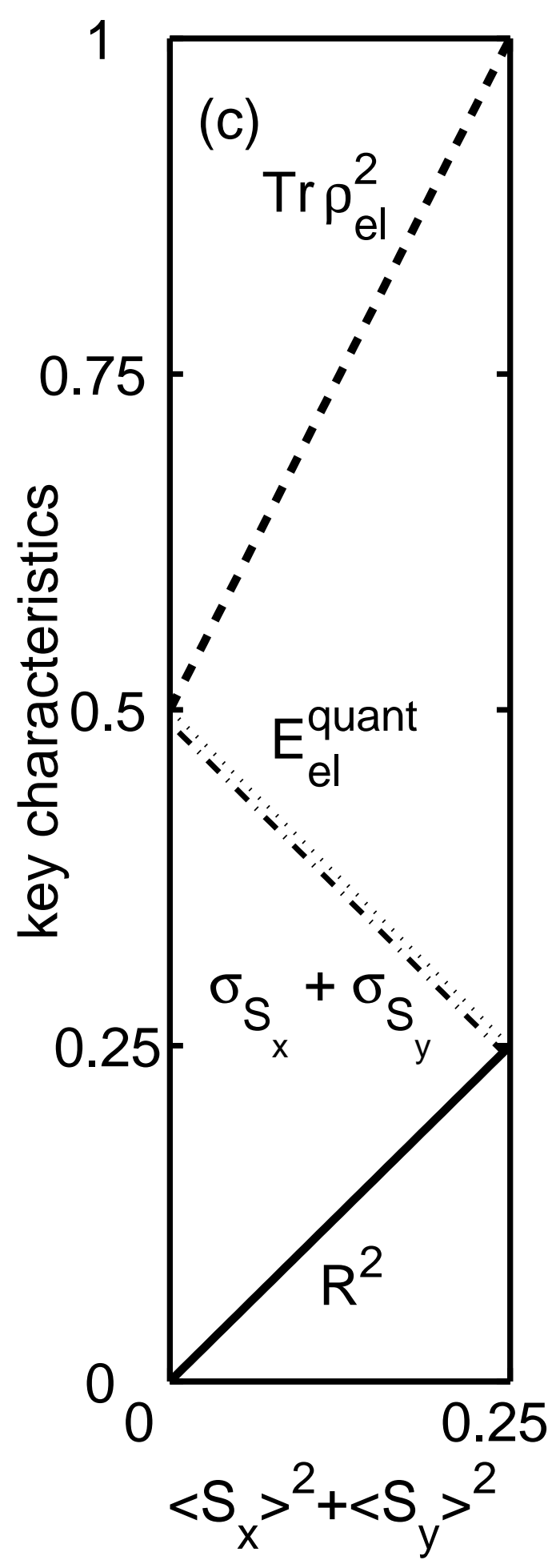

